A\&A 609, A113 (2018)

DOI: $10.1051 / 0004-6361 / 201730386$

(c) ESO 2018

\title{
Solar system science with ESA Euclid
}

\author{
B. Carry ${ }^{1,2}$ \\ 1 Université Côte d'Azur, Observatoire de la Côte d'Azur, CNRS, Lagrange, 06304 Nice, France \\ 2 IMCCE, Observatoire de Paris, PSL Research University, CNRS, Sorbonne Universités, UPMC Univ. Paris 06, Univ. Lille, France \\ e-mail: benoit.carry@oca.eu
}

Received 2 January 2017 / Accepted 3 November 2017

\begin{abstract}
Context. The ESA Euclid mission has been designed to map the geometry of the dark Universe. Scheduled for launch in 2020 , it will conduct a six-year visible and near-infrared imaging and spectroscopic survey over $15000 \mathrm{deg}^{2}$ down to $V_{\mathrm{AB}} \sim 24.5$. Although the survey will avoid ecliptic latitudes below $15^{\circ}$, the survey pattern in repeated sequences of four broadband filters seems well-adapted to detect and characterize solar system objects (SSOs).

Aims. We aim at evaluating the capability of Euclid of discovering SSOs and of measuring their position, apparent magnitude, and spectral energy distribution. We also investigate how the SSO orbits, morphology (activity and multiplicity), physical properties (rotation period, spin orientation, and 3D shape), and surface composition can be determined based on these measurements.

Methods. We used the current census of SSOs to extrapolate the total amount of SSOs that will be detectable by Euclid, that is, objects within the survey area and brighter than the limiting magnitude. For each different population of SSO, from neighboring near-Earth asteroids to distant Kuiper-belt objects (KBOs) and including comets, we compared the expected Euclid astrometry, photometry, and spectroscopy with the SSO properties to estimate how Euclid will constrain the SSOs dynamical, physical, and compositional properties.

Results. With the current survey design, about 150000 SSOs, mainly from the asteroid main-belt, should be observable by Euclid. These objects will all have high inclination, which is a difference to many SSO surveys that focus on the ecliptic plane. Euclid may be able to discover several $10^{4} \mathrm{SSOs}$, in particular, distant KBOs at high declination. The Euclid observations will consist of a suite of four sequences of four measurements and will refine the spectral classification of SSOs by extending the spectral coverage provided by Gaia and the LSST, for instance, to 2 microns. Combined with sparse photometry such as measured by Gaia and the LSST, the time-resolved photometry will contribute to determining the SSO rotation period, spin orientation, and 3D shape model. The sharp and stable point-spread function of Euclid will also allow us to resolve binary systems in the Kuiper belt and detect activity around Centaurs.

Conclusions. The depth of the Euclid survey ( $\left.V_{\mathrm{AB}} \sim 24.5\right)$, its spectral coverage $(0.5$ to $2.0 \mu \mathrm{m})$, and its observation cadence has great potential for solar system research. A dedicated processing for SSOs is being set up within the Euclid consortium to produce astrometry catalogs, multicolor and time-resolved photometry, and spectral classification of some $10^{5} \mathrm{SSOs}$, which will be delivered as Legacy Science.
\end{abstract}

Key words. methods: statistical - minor planets, asteroids: general - Kuiper belt: general - comets: general

\section{Introduction}

The second mission in ESA's Cosmic Vision program, Euclid is a wide-field space mission dedicated to the study of dark energy and dark matter through mapping weak gravitational lensing (Laureijs et al. 2011). It is equipped with a silicon-carbide $1.2 \mathrm{~m}$ aperture Korsch telescope and two instruments: a VISible imaging camera, and a Near Infrared Spectrometer and Photometer (VIS and NISP; see Cropper et al. 2014; Maciaszek et al. 2014). The mission design combines a large field of view (FoV, 0.57 $\operatorname{deg}^{2}$ ) with high angular resolution (pixel scales of $0.1^{\prime \prime}$ and $0.3^{\prime \prime}$ for VIS and NISP, corresponding to the diffraction limit at 0.6 and $1.7 \mu \mathrm{m})$.

Scheduled for a launch in 2020 and operating during six years from the Sun-Earth Lagrange L2 point, Euclid will carry out an imaging and spectroscopic survey of the extragalactic sky of $15000 \mathrm{deg}^{2}$ (the Wide Survey), avoiding galactic latitudes lower than $30^{\circ}$ and ecliptic latitudes below $15^{\circ}$ (Fig. 1), totaling 35000 pointings. A second survey, two magnitudes deeper and located at very high ecliptic latitudes, will cover $40 \mathrm{deg}^{2}$ spread across three areas (the Deep Survey). Additionally, 7000 observations of 1200 calibration fields, mainly located at $-10^{\circ}$ and $+10^{\circ}$ galactic latitude, will be acquired during the course of the mission to monitor the stability of the telescope point-spread function (PSF), and assess the photometric and spectroscopic accuracy of the mission.

Euclid imaging detection limits are required at $m_{\mathrm{AB}}=24.5$ $\left(10 \sigma\right.$ on a $1^{\prime \prime}$ extended source) with VIS, and $m_{\mathrm{AB}}=24(5 \sigma$ point source) in the $Y, J$, and $H$ filters with NISP. Spectroscopic requirements are to cover the same near-infrared wavelength range at a resolving power of 380 and to detect at $3.5 \sigma$ an emission line at $3 \times 10^{-16} \mathrm{erg} \mathrm{cm}^{-1} \mathrm{~s}^{-1}$ (on a $1^{\prime \prime}$ extended source). The NISP implementation consists of two grisms, red (1.25 to $1.85 \mu \mathrm{m})$ and blue (0.92 to $1.25 \mu \mathrm{m}$, usage of which will be limited to the Deep Survey), providing a continuum sensitivity to $m_{\mathrm{AB}} \approx 21$. To achieve these goals, the following survey operations were designed:

1. The observations will consist of a step-and-stare tiling mode, in which both instruments target the common $0.57 \mathrm{deg}^{2}$ FOV before the telescope slews to other coordinates.

2. Each tile will be visited only once, with the exception of the Deep Survey, in which each tile will be pointed at 40 times, 


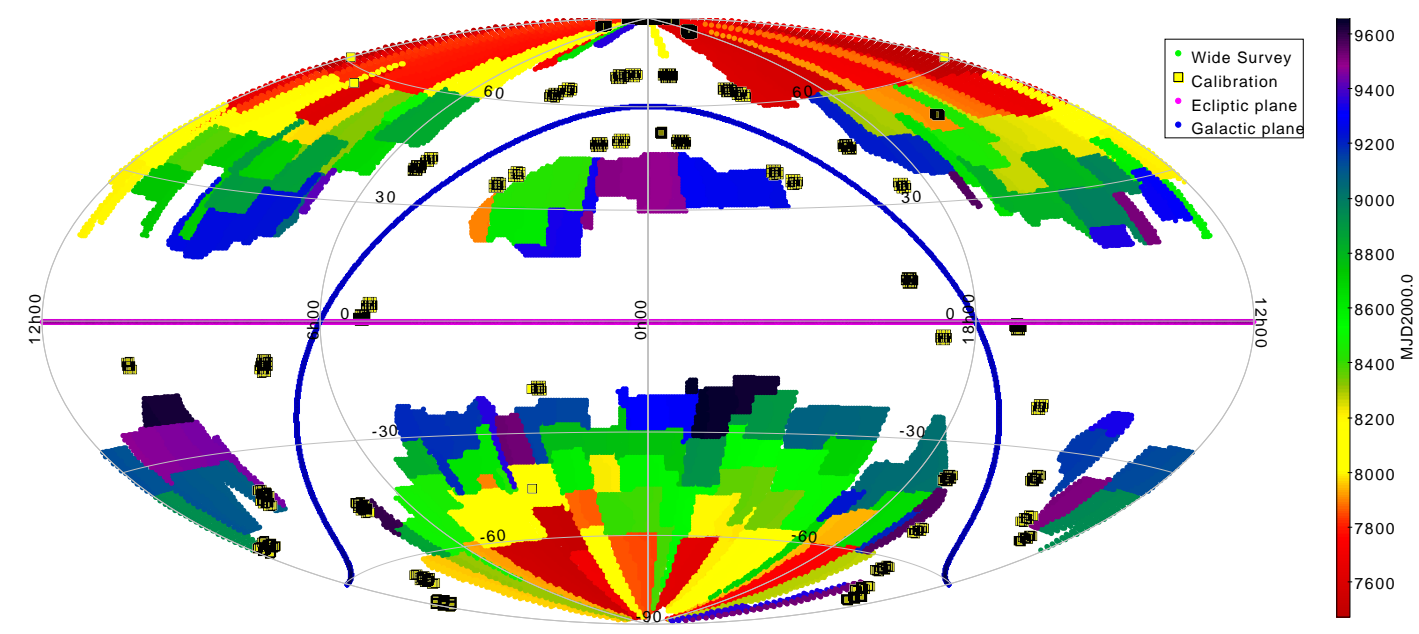

Fig. 1. Expected coverage of the Euclid Wide Survey (called the reference survey), color-coded by observing epoch, in an Aitoff projection of ecliptic coordinates. The horizontal gap corresponds to low ecliptic latitudes (the cyan line represents the ecliptic plane), and the circular gap to low galactic latitudes (the deep blue line stands for the galactic plane). The black squares filled with yellow are the calibration fields, which are to be repeatedly observed during the six years of the mission, to assess the stability and accuracy of the Euclid PSF, photometry, and spectroscopy.

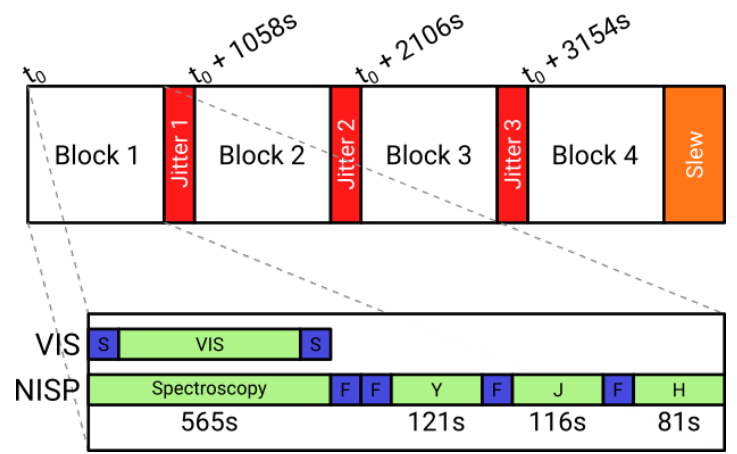

Fig. 2. Observation sequence for each pointing. The observing block, composed of a simultaneous VIS and NISP/spectroscopy exposure and three NISP/imaging exposures $(Y, J, H)$, is repeated four times, with small jitters $\left(100^{\prime \prime} \times 50^{\prime \prime}\right)$. The blue boxes $\mathrm{F}$ and $\mathrm{S}$ stand for overheads that are due to the rotation of the filter wheel and shutter opening/closure. Figure adapted from Laureijs et al. (2011).

and the calibration fields, which will be observed 5 times each on average.

3. The filling pattern of the survey will follow the lines of ecliptic longitude at quadrature. Current survey planning foresees a narrow distribution of the solar elongation of $\Psi=$ $91.0 \pm 1.5^{\circ}$ only; the range of solar elongation available to the telescope is limited to $87^{\circ}-110^{\circ}$.

4. The observation of each tile will be subdivided into four observing blocks that differ by only small jitters $\left(100^{\prime \prime} \times 50^{\prime \prime}\right)$. These small pointing offsets will allow to fill the gaps between the detectors that make up the focal plane of each instrument. In this way, $95 \%$ of the sky will be covered by three blocks, and $50 \%$ by four blocks.

5. In each block, near-infrared slitless spectra will be obtained with NISP simultaneously with a visible image with VIS, with an integration time of $565 \mathrm{~s}$. This integration time implies a saturation limit of $V_{\mathrm{AB}} \approx 17$ for a point-like source. Then, three NISP images will be taken with the $Y, J$, and $H$ near-infrared filters, with integration times of 121,116 , and $81 \mathrm{~s}$, respectively (Fig. 2).

All these characteristics make the Euclid survey a potential prime data set for legacy science. In particular, the access to the near-infrared sky, about seven magnitudes fainter than the DENIS and 2MASS (Epchtein et al. 1994; Skrutskie et al. 2006) surveys, and two to three magnitudes fainter than the current ESO VISTA Hemispherical Survey (VHS; McMahon et al. 2013), makes Euclid suitable for a surface characterization of solar system objects (SSOs), especially in an era rich in surveys that only operate in visible wavelengths, such as the Sloan Digital Sky Survey (SDSS), Pan-STARRS, ESA Gaia, and the Large Synoptic Sky Survey (LSST) (Abazajian et al. 2003; Jewitt 2003; Gaia Collaboration 2016; LSST Science Collaboration et al. 2009).

We discuss here the potential of the Euclid mission for solar system science. In the following, we consider the following populations of SSOs, defined by their orbital elements (Appendix A):

- near-Earth asteroids (NEAs), including the Aten, Apollo, and Amor classes, whose orbits cross the orbits of terrestrial planets;

- Mars-crossers (MCs), a transitory population between the asteroid main belt and near-Earth space;

- main-belt asteroids (MBA) in the principal reservoir of asteroids in the solar system, between Mars and Jupiter, split into Hungarian, inner main-belt (IMB), middle main-belt (MMB), outer main-belt (OMB), Cybele, and Hilda;

- Jupiter trojans (Trojans), orbiting the Sun at the Lagrange L4 and L5 points of the Sun-Jupiter system;

- Centaurs whose orbits cross the orbits of giant planets;

- Kuiper-belt objects (KBOs) farther away than Neptune, divided into detached, resonant, and scattered-disk objects (SDO), and inner, main, and outer classical belt (ICB, MCB, and $\mathrm{OCB}$ ); and

- comets from the outskirts of the solar system on highly eccentric orbits that are characterized by activity (coma) at short heliocentric distances.

The discussion is organized as follows: the expected number of SSO observations is presented in Sect. 2, and the difficulties we expect for these observations are described in Sect. 3. The problems of source identification and the contribution to astrometry and orbit determination are discussed in Sect. 4. Then the potential for spectral characterization from VIS and NISP photometry is detailed in Sect. 5, and the same is done for 

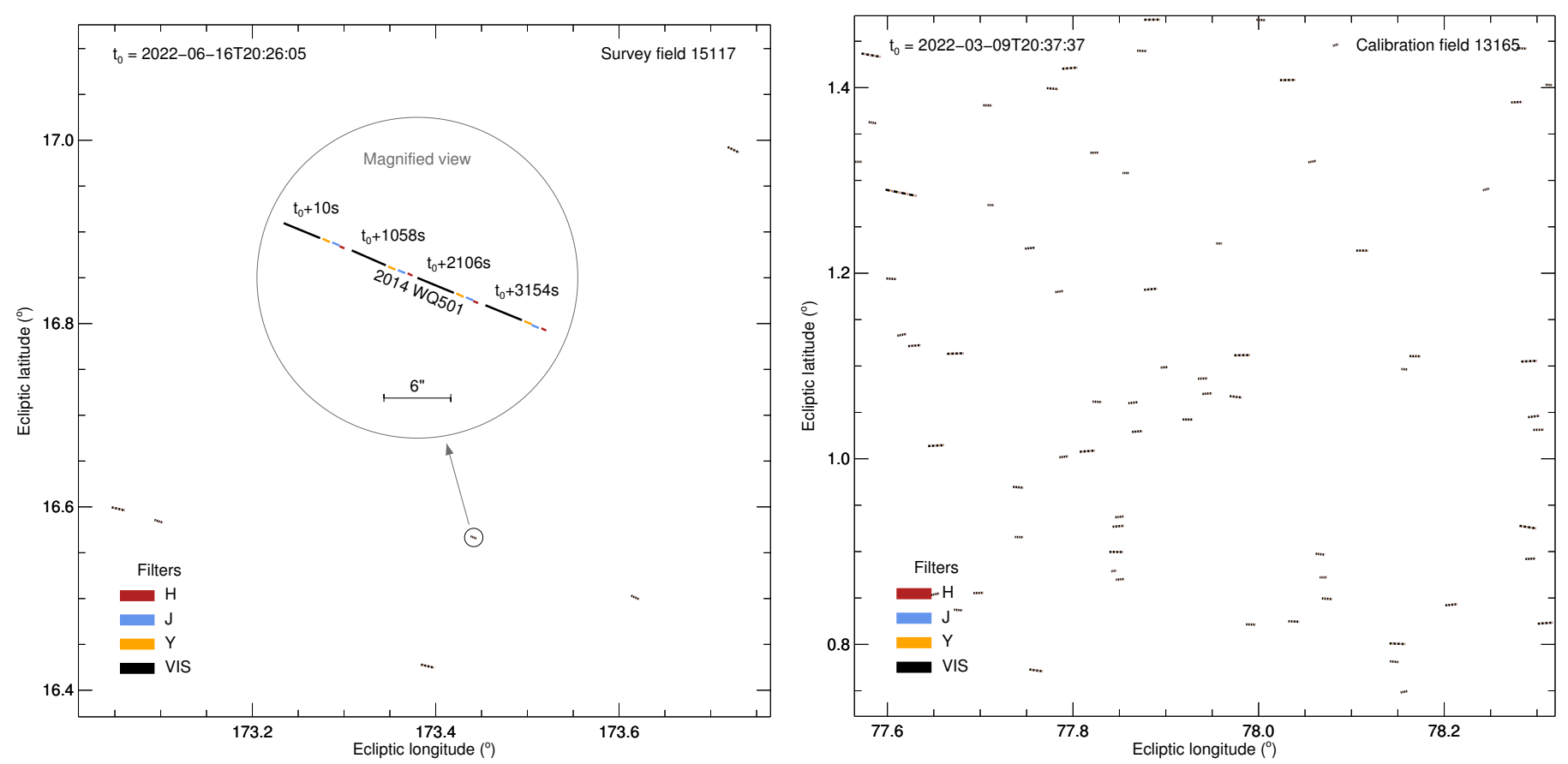

Fig. 3. Examples of the contamination of Euclid FOV by SSOs. Left: survey field 15117 centered on (RA, Dec) $=\left(167.218^{\circ},+12.740^{\circ}\right)$ and starting on 2022 June 16 at 20:26:05 UTC. The successive trails impressed by the 6 known SSOs during the Euclid hour-long sequence of VIS-NISP imaging observations are drawn in different colors, one for each filter (VIS, $Y, J$, and $H$ ). We can expect about a hundred times more SSOs at the limiting magnitude of Euclid (e.g., Fig. 4). The inset is a magnified view of 2014 WQ501, a main-belt asteroid, illustrating the highly elongated shape of an SSO in Euclid frames. The scale bar of 6" corresponds to 60 pixels in VIS frames and 20 pixels in NISP. The timings reported are the starting time of the VIS exposures. The slitless spectra will be acquired by NISP simultaneously with the VIS images. Right: calibration field 13165 centered on $(\mathrm{RA}, \mathrm{Dec})=\left(76.785^{\circ},+23.988^{\circ}\right)$ and starting on $2022 \mathrm{March} 9$ at 20:37:37 UTC. There are 117 known SSOs in the field, and here also, a hundred times more SSOs will be detected at the limiting magnitude of Euclid.

NISP spectroscopy in Sect. 6. The Euclid capabilities for directly imaging satellites and SSO activity are presented in Sect. 7, and the contribution of Euclid to the 3D shape and binarity modeling from light curves is described in Sect. 8.

\section{Expected number of SSO observations}

Although the Euclid Wide survey will avoid the ecliptic plane (Fig. 1), its observing sequence is by chance well adapted to detect moving objects. As described above, each FoV will be imaged 16 times in one hour in four repeated blocks. Given the pixel scale of the VIS and NISP cameras of $0.1^{\prime \prime}$ and $0.3^{\prime \prime}$, any SSO with an apparent motion higher than $\approx 0.2^{\prime \prime} / \mathrm{h}$ should therefore be detected by its trailed appearance and/or motion across the different frames (Fig. 3).

To estimate the number of SSOs that might be detected by Euclid, we first built the cumulative size distribution (CSD) of each population. We used the absolute magnitude $H$ as a proxy for the diameter $D$. The relation between these two is $D(\mathrm{~km})=1329 p_{V}^{-1 / 2} 10^{-0.2 H}$ (e.g., Bowell et al. 1989), where $p_{V}$ is the surface albedo in $\mathrm{V}$, which quantifies its capability of reflecting light. Minor planets, especially asteroids, tend to be very dark, and their albedo is generally very low, from a few percents to $\approx 30 \%$ (see, e.g., Mainzer et al. 2011).

We retrieved the absolute magnitude from the astorb database (Bowell et al. 1993), with the exception of comets, which are not listed in astorb, and for which we used the compiled data by Snodgrass et al. (2011). The challenge was then to extrapolate the observed distributions (shown as solid lines in Fig. 4) to smaller sizes. Most are close to power-law distributions (Dohnanyi 1969) in the form $\mathrm{d} N / \mathrm{d} H \propto 10^{\gamma H}$, with different slopes $\gamma$. We model each population below and represent them with dashed lines in Fig. 4:

- NEAs: we used the synthetic population by Granvik et al. (2016), which is very similar to the population used by Harris \& D'Abramo (2015). However, we took a conservative approach and increased the uncertainty of the model to encompass both estimates.

- MCs: no dedicated study of the CSD of MCs is available. We therefore took the NEA model above, scaled by a factor of three, to match the currently known MC population. The upper estimate was taken as a power-law fit to the current population with $\gamma=0.41$, and the lower estimate is that of the scaled NEA model by Granvik et al. (2016), reduced by a factor of two.

- MBAs: we used the knee distribution by Gladman et al. (2009), in which large objects $(H \in[11,15])$ follow a steep slope $(\gamma \sim 0.5)$, while smaller asteroids follow a shallower slope of $\gamma=0.30 \pm 0.02$ in the range $H \in[15,18]$, after which no constraint is available. This model is scaled to 25954 asteroids at $H=15$. These authors found the CSD to be very smooth in this absolute magnitude range compared to earlier works (Jedicke \& Metcalfe 1998; Ivezić et al. 2001; Wiegert et al. 2007). We modified their model only slightly by changing the slope at $H=15.25$ instead of $H=15$ : the shallower slope does no longer fit the observed data below $H=15.25$. The observing strategy by Gladman et al. (2009) was indeed aimed at constraining the faint end of the CSD, and the constraints on large bodies was weak (only a small sky area had been targeted).

- Trojans: we used the model of Jewitt et al. (2000), with $\gamma=0.4 \pm 0.06$. More recently, Grav et al. (2011) found 


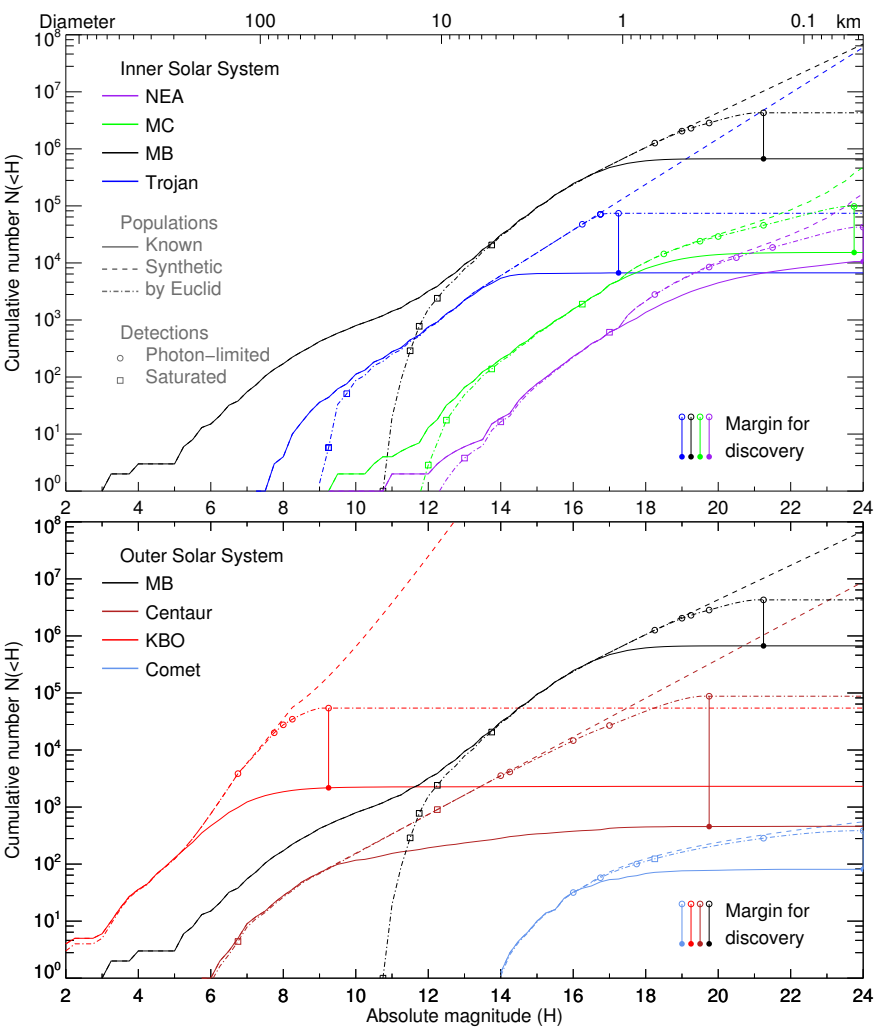

Fig. 4. Cumulative size distribution of each SSO population for current census (solid lines) and synthetic populations (average estimates represented by the dashed lines, upper and lower estimate not plotted for clarity). The number of known objects that is observable at the limiting apparent magnitude of Euclid over the entire celestial sphere is represented by the dot-dashed lines. The open squares and circles represent the $0,25,50,75$, and $100 \%$ marks of the $(H-V)$ cumulative probability function at the saturation and photon-starving ends. The total number of objects expected on the sky is marked by the filled circles. The difference between these filled circles and the current census represents the margin for discovery.

a similar $\gamma$, but restricted their study to Trojans with $D>$ $10 \mathrm{~km}$. We scaled their model to the number of 310 known Trojans at $H=12.5$. The steeper slope (i.e., $\gamma=0.46$ ) seems to reproduce the currently observed population more accurately. The baseline numbers for Trojans presented here may therefore be underestimated, and the upper estimate might represent the real Trojan population better. Finally, we did not use the knee model by Yoshida \& Nakamura (2005), who predicted a change in slope at $D \approx 5 \mathrm{~km}$, because their model no longer fits the known population.

- Centaurs: we used the $\gamma=0.34 \pm 0.04$ from Bauer et al. (2013), which is close to the 0.4 estimate from Jedicke et al. (2002). We scaled the power law to correspond to the cumulative population (7) at $H=8.25$.

- KBOs: first, we built the CSD of the resonant population using a single power law of index $\gamma=0.9_{-0.4}^{+0.2}$, scaled to a total of 22000 objects a $H=8.66$, as proposed by Volk et al. (2016) based on the early results of the Outer Solar System Origins Survey (OSSOS; Bannister et al. 2016), which is consistent with the earlier work by Gladman et al. (2012) based on the Canada-France Ecliptic Plane Survey (CFEPS). Then, we built the CSD of the scattered-disk objects using the divot distribution by Shankman et al. (2016): large objects follow a steep slope $(\gamma \sim 0.9)$, scaled to a total of 6500 objects a $H=8$, which changes at $H=8.0$ to a shallower $\gamma=0.50_{-0.08}^{+0.15}$. The differential size distribution presents a drop at $H \stackrel{-08}{=} 8.0$ where the slope changes, the smaller objects being less numerous by a factor of 5.6 (see Shankman et al. 2016, for details). Finally, we took the CSD of objects in the classical belt from Petit et al. (2016), who proposed a knee distribution: $\gamma=1.02$, scaled to a total of 1800 objects at $H=7$, until $H=7.0$ (in agreement with Adams et al. 2014), where it switches to $\gamma=0.65 \pm 0.05$. The CSD for the entire KBO population is the sum of the three aforementioned CSD.

- Comets: we used the knee CSD from Snodgrass et al. (2011). Largest comets follow a $\gamma=0.38_{-0.04}^{+0.06}$ until $H=17$ (converted from the turnover radius of $1.25 \mathrm{~km}$ using an albedo of 0.04), after which the CSD is shallower, although less constrained, and we assumed the average slope found by Snodgrass et al. (2011) with arbitrary uncertainties: $\gamma=0.04_{-0.02}^{+0.06}$.

The question is then which range of absolute magnitudes will be accessible to Euclid for each population, considering it will observe in the range $V_{\mathrm{AB}}=17-24.5$. This conversion from apparent to absolute magnitude only depends on the geometry of observation (Bowell et al. 1989) through the heliocentric distance $(\Delta)$, range to the observer $(r)$, and phase angle $(\alpha$, the angle between the target-Sun and target-observer vectors):

$H=V+2.5 \log \left(r^{2} \Delta^{2}\right)-2.5 \log \left((1-G) \phi_{1}+G \phi_{2}\right)$,

with the phase functions approximated by

$\phi_{1}=\exp \left(-3.33 \tan \left(\frac{\alpha}{2}\right)^{0.63}\right)$
$\phi_{2}=\exp \left(-1.87 \tan \left(\frac{\alpha}{2}\right)^{1.22}\right)$.

Although a more accurate model (with two phase slopes $G_{1}$ and $G_{2}$ ) of the phase dependence has been developed recently (Muinonen et al. 2010), the differences in the predicted magnitudes between the two systems are minor for our purpose. We therefore use the former and simpler $\mathrm{H}-\mathrm{G}$ system in the following, assuming the canonic value of $G=0.15$.

The three geometric parameters $(r, \Delta, \alpha)$ are tied together by the solar elongation $\Psi$, which is imposed by the spacecraft operations $\left(\Psi=91.0 \pm 1.5^{\circ}\right)$. In practice, it is sufficient to estimate the range of heliocentric distances at which Euclid will observe an SSO from a given population to derive the two other geometric quantities, and hence the $(H-V)$ index:

$$
\begin{aligned}
r & =\cos \Psi+\sqrt{\cos ^{2} \Psi-1+\Delta^{2}} . \\
\alpha & =\left|\operatorname{asin}\left(\frac{\sin \Psi}{\Delta}\right)\right| .
\end{aligned}
$$

We thus computed the probability density function (PDF) of the heliocentric distance of each population. For this, we computed the 2D distribution of the semimajor axis $v s$ eccentricity of each population using bins of 0.05 in AU and eccentricity. For each bin, we computed the PDF of the heliocentric distance from Kepler's second law. We then summed individual PDFs from each bin, normalized by the number of SSOs in each bin divided by the entire population.

We then combined the distribution of the solar elongation from the reference survey and the PDF of the heliocentric distance of each population in Eqs. (4) and (5) to obtain a PDF of the $(\mathrm{H}-\mathrm{V})$ index (Eq. (1)). The fraction of populations to be observed by Euclid at each magnitude is estimated by multiplying 
Table 1. Expected number of SSOs observed by Euclid for each population.

\begin{tabular}{lrcrcccccc}
\hline \hline \multicolumn{2}{c}{ Population } & \multicolumn{1}{c}{ All-sky } & \multicolumn{1}{c}{$f_{\mathrm{W}}$} & \multicolumn{2}{c}{$f_{\mathrm{C}}$} & \multicolumn{3}{c}{ Euclid } & \multicolumn{3}{c}{ Absolute magnitude limits } \\
Name & \multicolumn{1}{c}{$\mathcal{N}_{\text {now }}$} & \multicolumn{1}{c}{$\mathcal{N}_{\mathrm{S}}$} & \multicolumn{1}{c}{$(\%)$} & \multicolumn{1}{c}{$(\%)$} & \multicolumn{1}{c}{$\mathcal{N}_{\mathrm{E}, \mathrm{d}}$} & \multicolumn{1}{c}{$\mathcal{N}_{\mathrm{E}, \mathrm{o}}$} & \multicolumn{1}{c}{$H_{100}$} & \multicolumn{1}{c}{$H_{50}$} & \multicolumn{1}{c}{$H_{1}$} \\
\hline NEA & 16062 & $1.9_{-0.6}^{+1.1} \times 10^{5}$ & $7.2 \pm 0.4$ & $0.8 \pm 0.1$ & $1.4_{-0.5}^{+1.0} \times 10^{4}$ & $1.5_{-0.6}^{+1.0} \times 10^{4}$ & 22.75 & 23.75 & 26.50 \\
MC & 15488 & $1.2_{-0.8}^{+1.6} \times 10^{5}$ & $9.0 \pm 0.6$ & $0.6 \pm 0.1$ & $1.0_{-0.8}^{+1.7} \times 10^{4}$ & $1.2_{-0.8}^{+1.7} \times 10^{4}$ & 21.00 & 21.25 & 22.75 \\
MB & 674981 & $4.3_{-0.9}^{+1.0} \times 10^{6}$ & $1.5 \pm 0.0$ & $0.7 \pm 0.0$ & $8.2_{-2.2}^{+2.5} \times 10^{4}$ & $9.7_{-2.2}^{+2.5} \times 10^{4}$ & 19.50 & 20.00 & 21.25 \\
Trojan & 6762 & $1.3_{-0.7}^{+0.9} \times 10^{5}$ & $5.1 \pm 1.5$ & $0.5 \pm 0.4$ & $7.1_{-4.9}^{+9.3} \times 10^{3}$ & $7.5_{-5.0}^{+9.5} \times 10^{3}$ & 17.00 & 17.25 & 18.25 \\
Centaur & 470 & $1.8_{-1.0}^{+1.4} \times 10^{4}$ & $12.2 \pm 0.9$ & $0.6 \pm 0.4$ & $2.2_{-1.4}^{+2.1} \times 10^{3}$ & $2.2_{-1.4}^{+2.1} \times 10^{3}$ & 14.75 & 15.50 & 18.25 \\
KBO & 2331 & $9.8_{-1.9}^{+2.2} \times 10^{4}$ & $4.9 \pm 0.2$ & $0.6 \pm 0.1$ & $5.3_{-1.3}^{+1.6} \times 10^{3}$ & $5.5_{-1.3}^{+1.6} \times 10^{3}$ & 8.25 & 8.75 & 10.00 \\
Comet & 1301 & $185.2_{-13.5}^{+15.4}$ & $19.5 \pm 0.5$ & $1.0 \pm 0.3$ & $21.5_{-3.6}^{+4.2}$ & $38.2_{-4.3}^{+4.9}$ & 18.25 & 19.00 & 22.00 \\
\hline Total & 717395 & $4.9_{-1.2}^{+1.4} \times 10^{6}$ & $2.1 \pm 0.1$ & $0.7 \pm 0.0$ & $1.2_{-0.4}^{+0.7} \times 10^{5}$ & $1.4_{-0.4}^{+0.7} \times 10^{5}$ & & & \\
\hline
\end{tabular}

Notes. For the whole celestial sphere, we report the current number of known SSOs $\left(\mathcal{N}_{\text {now }}\right.$, at the time of the writing on 2017 June 28$)$, the expected number of observable objects $\left(\mathcal{N}_{\mathrm{S}}\right)$ at the limiting apparent magnitude of Euclid $\left(V_{\mathrm{AB}}<24.5\right)$, and the solar elongation $\left(\Psi=91.0 \pm 1.5^{\circ}\right)$. Using the fraction of known SSOs within the area of the Euclid Wide survey $\left(f_{\mathrm{W}}\right)$ and calibration frames $\left(f_{\mathrm{C}}\right)$, we estimate the total number of discoveries $\left(\mathcal{N}_{\mathrm{E}, \mathrm{d}}\right)$ and observations $\left(\mathcal{N}_{\mathrm{E}, \mathrm{o}}\right)$ by Euclid. The absolute magnitude corresponding to a probability of $100 \%, 50 \%$, and $1 \%$ that SSOs will be within the detection envelop of Euclid are also reported.

the CSD of the synthetic populations with the cumulative distribution of the $(\mathrm{H}-\mathrm{V})$ index at either end of the magnitude range of Euclid $\left(V_{\mathrm{AB}}=17-24.5\right.$, see the dot-dashed lines in Fig. 4$)$. The number of observable SSOs on the entire celestial sphere $\left(\mathcal{N}_{\mathrm{S}}\right)$ can be read from this graph, and they are reported in Table 1. The difference between synthetic and observed population also provides an estimate of the potential number of objects to be discovered by Euclid down to $V_{\mathrm{AB}}=24.5$.

We then estimated how many of these objects will be observed by Euclid. For this, we computed the position of all known SSOs every six months for the entire duration of the Euclid operations (2020 to 2026) using the Virtual Observatory (VO) web service SkyBot 3D ${ }^{1}$ (Berthier et al. 2008). This allows computing the fraction of known SSOs within the area covered by the Euclid surveys $\left(f_{\mathrm{W}}, f_{\mathrm{D}}\right.$, and $f_{\mathrm{C}}$ for the Wide and Deep Surveys, and calibration frames). We report these fractions in Table 1, except for $f_{\mathrm{D}}$, which is negligible (on the order of 1-10 ppm) because only very few SSOs on highly inclined orbits are known (although there is a clear bias against discovering such objects in the current census of SSOs, see Petit et al. 2017; Mahlke et al. 2017). These figures are roughly independent of the epoch for all populations but for the Trojans, which are confined around the Lagrangian L4 and L5 points on Jupiter's orbit and therefore cover a limited range in right ascension at each epoch.

Overall, about 150000 SSOs are expected to be observed by Euclid in a size range that is currently unexplored by large surveys. This estimate may be refined once dedicated studies of the detection envelop of moving objects will be performed on simulated data. Euclid could discover thousands of outer SSOs and tens of thousands of sub-kilometric main-belt, Mars-crosser, and near-Earth asteroids (see the typical absolute magnitudes probed by Euclid in Table 1). Nevertheless, the Large Synoptic Survey Telescope (LSST, LSST Science Collaboration et al. 2009) is expected to see scientific first-light in 2021. The LSST will repeatedly image the sky down to $V \approx 24$ over a wide range of solar elongations, and will be a major discoverer of faint SSOs. Assuming a discovery rate of 10000 NEAs, $10000 \mathrm{MCs}$, 550000 MBAs, 30000 Trojans, 3000 Centaurs, 4000 KBOs, and 1000 comets per year (LSST Science Collaboration et al. 2009), most of the SSOs that are potentially available for discovery

\footnotetext{
1 http://vo.imcce.fr/webservices/skybot3d/
}

are expected to be discovered by the LSST in the southern hemisphere. The exploration of small KBOs in the northern hemisphere will be reserved for Euclid, however.

\section{Specificity of the SSO observations with Euclid}

The real challenge of SSO observations with Euclid will be the astrometry and photometry of highly elongated sources (as indicated in Fig. 3). We present in Fig. 5 and Table 2 a summary of the apparent non-sidereal rate of the different SSO populations. With the exception of the most distant populations of KBOs, Centaurs, and comets, all SSOs will present rates above $10^{\prime \prime} / \mathrm{h}$. This implies a motion of hundreds of pixels between the first and last VIS frame. During a single exposure, each SSO will move and produce a trailed signature, a streak, whose length will typically range from 1 to 50 pixels for VIS. The situation will be more favorable for NISP because of the shorter integration times and larger pixel scale, and most SSOs will not trail, or will only trail across a few pixels (Table 2).

Some recent developments have been made to detect streaks, motivated by the optical detection and tracking of artificial satellites and debris on low orbits around the Earth. Dedicated image processing for trails can be set up to measure the astrometry and photometry of moving objects within a field of fixed stars, without an a priori knowledge of their apparent motion (e.g., Virtanen et al. 2016). The success rate in detecting these trails has been shown to reach up to $90 \%$, even in the regime with low signal-to-noise ratio $(\approx 1)$. These algorithms are currently being tested on simulated Euclid data of SSOs (M. Granvik, priv. comm.).

\section{Source identification, astrometry, and dynamics}

As established in Sect. 2, Euclid will observe about 150000 SSOs, even if its nominal survey avoids ecliptic latitudes below $15^{\circ}$, with the notable exception of the calibration fields (Fig. 1).

The design of the surveys, with hour-long sequences of observation of each field, will preclude orbit determination for newly discovered objects, however. This hour-long coverage is nevertheless sufficient to distinguish between NEAs, MBAs, and 


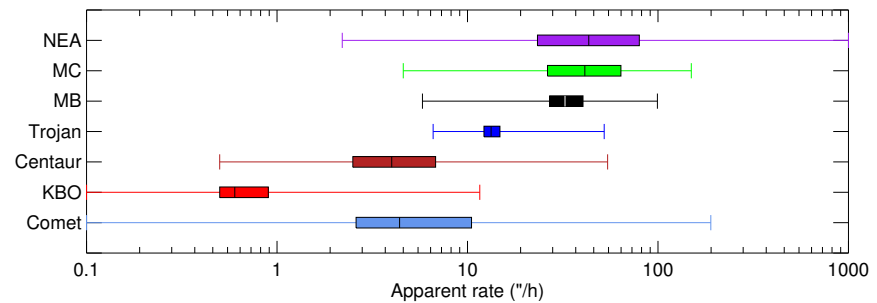

Fig. 5. Five-number summary (minimum, maximum, median, $25 \%$, and $75 \%$ quartiles) of the apparent rate of each SSO population. The Euclid mode of observation at quadrature reduces the apparent rate compared to opposition, for example.

Table 2. Apparent motion and trailing of SSOs observed by Euclid.

\begin{tabular}{lrrcccc}
\hline \hline Population & $\begin{array}{r}\text { Rate } \\
(\text { ('/h) }\end{array}$ & $\begin{array}{c}\text { VIS } \\
\text { (pix) }\end{array}$ & $\begin{array}{c}\text { NISP } \\
\text { (pix) }\end{array}$ & $\begin{array}{c}Y \\
\text { (pix) }\end{array}$ & $\begin{array}{c}J \\
\text { (pix) }\end{array}$ & $\begin{array}{c}H \\
(\text { pix })\end{array}$ \\
\hline NEA & $43.3_{-19.9}^{+36.5}$ & 67.9 & 22.6 & 4.8 & 4.6 & 3.2 \\
MC & $41.3_{-14.9}^{+22.6}$ & 64.8 & 21.6 & 4.6 & 4.4 & 3.1 \\
MB & $32.5_{-5.5}^{+7.9}$ & 51.0 & 17.0 & 3.6 & 3.5 & 2.4 \\
Trojan & $13.3_{-1.1}^{+1.4}$ & 20.9 & 7.0 & 1.5 & 1.4 & 1.0 \\
Centaur & $4.0_{-1.5}^{+2.9}$ & 6.2 & 2.1 & 0.4 & 0.4 & 0.3 \\
KBO & $0.6_{-0.1}^{+0.3}$ & 1.0 & 0.3 & 0.1 & 0.1 & 0.0 \\
Comet & $4.4_{-1.8}^{+6.2}$ & 6.9 & 2.3 & 0.5 & 0.5 & 0.3 \\
\hline
\end{tabular}

Notes. For each main population of SSOs, we report the apparent rate and its $25 \%$ and $75 \%$ quartile variations (i.e., half the population is within these two values), and the length of the trails on the detector during the simultaneous VIS imaging and NISP spectroscopic $565 \mathrm{~s}$ exposure, and the following NISP $Y, J$, and $H$ imaging exposures of 121 , 116 , and $81 \mathrm{~s}$.

KBOs (Spoto et al. 2017). The situation will be very similar to that of the SDSS Moving Object Catalog (MOC), in which many SSO sightings corresponded to unknown objects at the time of the release (still about $53 \%$ at the time of the fourth release, Ivezić et al. 2001, 2002). Attempts at identification will have to be regularly performed a posteriori when the number of known objects, hence orbits, will increase, in the same way as we did for the SDSS MOC, where we identified $27 \%$ of the unknown sources (Carry et al. 2016) using the SkyBoT Virtual Observatory tool (Berthier et al. 2006, 2016). The success rate for an a posteriori identification of SSOs detected by Euclid is expected to be even higher than in this last study, as the LSST will be sensitive to the same apparent magnitude range.

Compared with tens of points over many years provided by the LSST, the astrometry by Euclid will probably contribute little to the determination of SSO orbits, with the following exceptions. First, the objects in the outer solar system (Centaurs and KBOs) in the northern hemisphere will not be observed by the LSST. In this respect, the Deep Survey will allow us to study the population of highly inclined Centaurs and KBOs (e.g., Petit et al. 2017) through repeated observations of the northern Ecliptic cap (about 40 times). Second, the parallax between the Earth and the Sun-Earth L2 point is large, from about a degree for asteroids in the inner belt to a few tens of arcseconds for KBOs. Simultaneous observation of the same field from the two locations thus provides the distance of the SSO, which drastically reduces the possible orbital parameter space (Eggl 2011). Thus, an interesting synergy between the LSST and Euclid will lie in planning these simultaneous observations (see Rhodes et al. 2017). The practical implementation may be difficult, however, as the observations by Euclid at a solar elongation $\Psi$ of $91.0 \pm 1.5^{\circ}$ impose observations close to sunset or sunrise from the LSST.

\section{Photometry and spectral classification}

In this section we study the effect of Euclid on the spectral classification of SSOs through determining their spectral energy distribution (SED; see Appendix B) over a wide wavelength range, from the visible with VIS $(0.5 \mu \mathrm{m})$ to the nearinfrared with NISP $(2 \mu \mathrm{m})$. While colors in the visible have been and will be obtained for several $10^{6}$ SSOs through surveys such as ESA Gaia and the LSST (Gaia Collaboration 2016; LSST Science Collaboration et al. 2009), a collection of nearinfrared photometry is lacking. The only facility currently operating from which near-infrared colors for numerous SSOs have been obtained is the ESO VISTA telescope (Popescu et al. 2016). As described above, the upcoming ESA Euclid mission (and also the NASA WFIRST mission, which shares many specifications with Euclid, see Green et al. 2012; Holler et al. 2017) may radically change this situation.

At first order, SSOs display a G2V spectrum at optical wavelength because the light of the Sun is reflected by their surface. Depending on their surface composition, regolith packing, and degree of space weathering, their spectra are modulated by absorption bands and slope effects, however. Historically, SSOs spectra have always been studied in reflectance, that is, their recorded spectrum divided by the spectrum of the Sun, approximated by a G2V star observed with the same instrument setting as the scientific target. The colors and low resolution ( $R \approx 300-500$ ) of asteroids have been used for decades to classify them in a scheme called taxonomy, which only uses the visible range or only the near-infrared, or both (see Chapman et al. 1975; Barucci et al. 1987; Bus \& Binzel 2002b,a; DeMeo et al. 2009). For KBOs, broadband colors and medium resolution $(R \approx$ 3000-5000) have been used to characterize their surface composition (e.g., Snodgrass et al. 2010; Carry et al. 2011, 2012), although current taxonomy is based on broadband colors alone (Fulchignoni et al. 2008).

Information on the taxonomic class has been derived for about 4000 asteroids based on their low-resolution spectra (mainly from the SMASS, SMASSII, and $\mathrm{S}^{3} \mathrm{OS}^{2}$ surveys, see Bus \& Binzel 2002b,a; Lazzaro et al. 2004). Using the broadband photometry from the Sloan Digital Sky Survey (SDSS), many studies have classified tens of thousands of asteroids (e.g., Ivezić et al. 2001, 2002; Nesvorný et al. 2005; Carvano et al. 2010; DeMeo \& Carry 2013). These studies opened a new era in the study of asteroid families (Carruba et al. 2013), space weathering (Nesvorný et al. 2005; Thomas et al. 2012), in the distribution of material in the inner solar system (DeMeo \& Carry 2014; DeMeo et al. 2014), and in the origins of near-Earth asteroids (Carry et al. 2016). The ongoing survey ESA Gaia will provide low-resolution spectra $(R \approx 35)$ for 300000 asteroids with high photometric accuracy, and the taxonomic class will be determined for each SSO (Delbo et al. 2012).

Nevertheless, any classification based on SDSS, Gaia, or the LSST (which will use a filter set comparable to that of SDSS), suffers from a wavelength range limited to only the visible. It is known, however, that several classes are degenerate over this spectral range, and only near-infrared colors/spectra can distinguish them (Fig. 6 and DeMeo et al. 2009). The near-infrared photometry provided by Euclid will therefore be highly valuable, similar to that reported from the 2MASS (Sykes et al. 2000) or ESO VISTA VHS (McMahon et al. 2013; Popescu et al. 2016) surveys. 


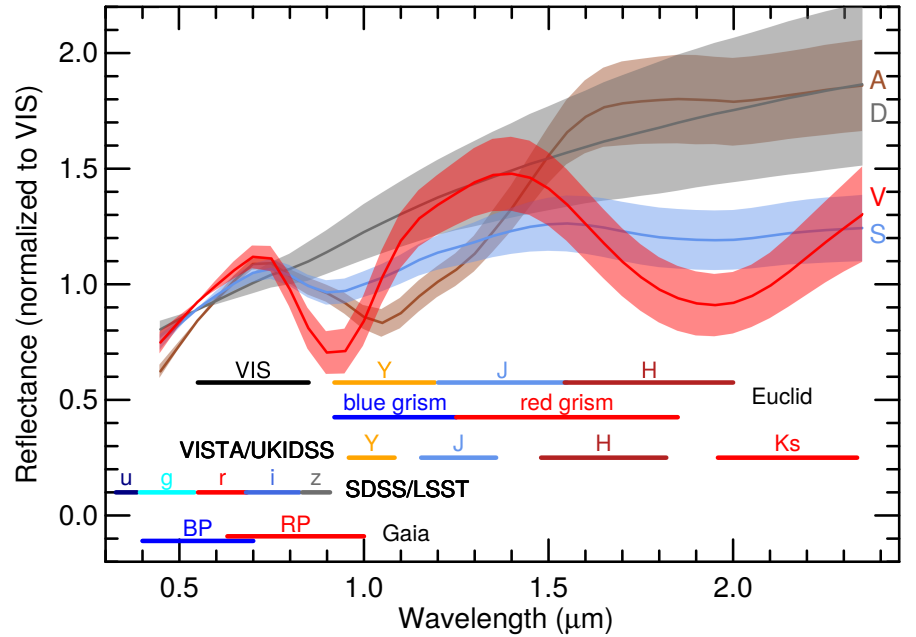

Fig. 6. Examples of asteroid classes (A, D, S, and V) that are degenerate over the visible wavelength range. For reference, the wavelength coverage of each photometric filter and grism on board Euclid is shown, together with the filter sets of SDSS and LSST $(u, g, r, i, z$; Ivezić et al. 2001), VISTA and UKIDSS ( $Y, J, H, K$ s; Hewett et al. 2006), and the Gaia blue and red photometers (BP, RP) that will produce low-resolution spectra (resolving power of a few tens; Delbo et al. 2012).

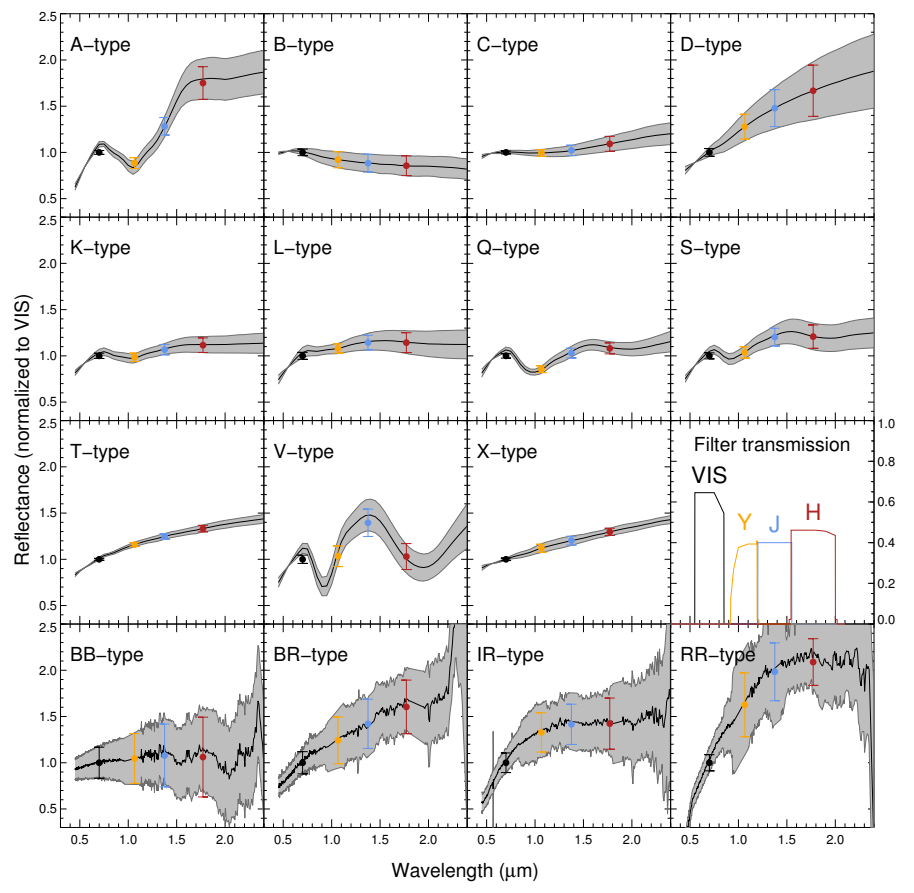

Fig. 7. Eleven asteroid (A- to X-type) and four $\mathrm{KBO}$ (BB, BR, IR, $\mathrm{RR}$ ) spectral classes considered here, converted into photometry for the classification simulation (see text). The transmission curves of the VIS and NISP filters are also plotted for reference.

To estimate the potential of the Euclid photometry for a spectral classification of asteroids, we simulated data using the visible and near-infrared spectra of the 371 asteroids that were used to create the Bus-DeMeo taxonomy (DeMeo et al. 2009), and of 43 KBOs with known taxonomy (Merlin et al. 2017). We converted their reflectance spectra into photometry (Fig. 7), taking the reference VIS and NISP filter transmission curves ${ }^{2}$.

\footnotetext{
2 Available at the Geneva university Euclid pages.
}

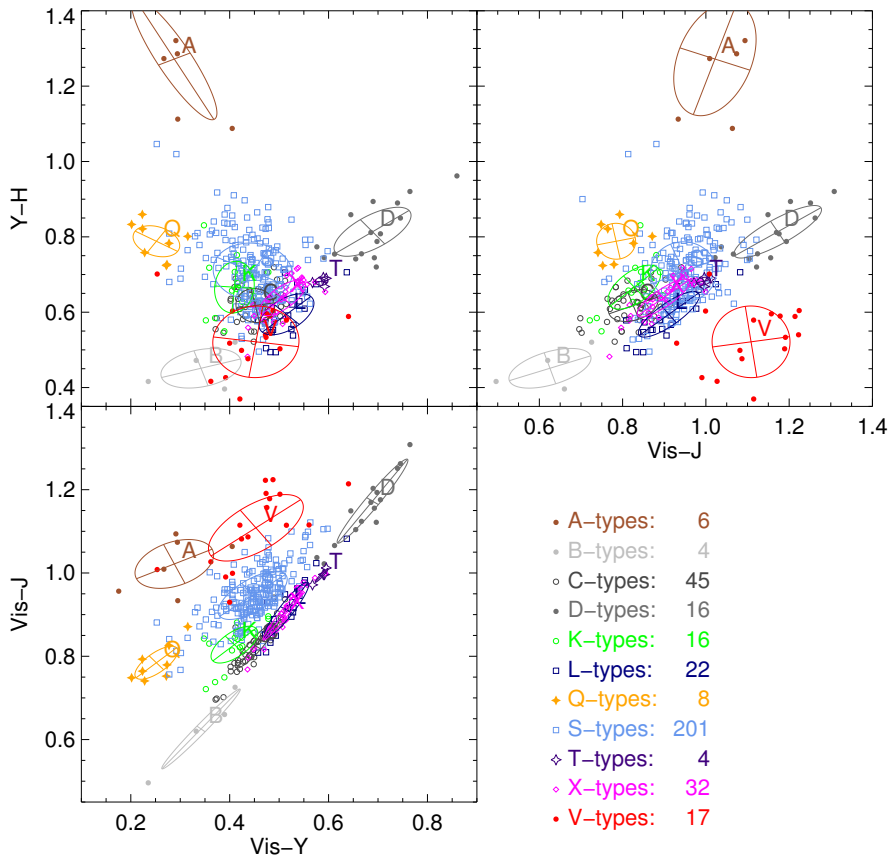

Fig. 8. Classification results for the 371 asteroids from Bus-DeMeo taxonomy, presented in three filter combinations: VIS-Y, VIS-J, and Y-H. Several extreme classes, such as A, B, D, V, and T, can be easily discarded thanks to the large wavelength coverage of Euclid.

One key aspect of the Euclid operations in determining the SSO colors is the repetition of the four-filter sequence during an hour. Thus, each filter will be bracketed by other filters in time. This will allow determining magnitude difference between each pair of filters without the biases that are otherwise introduced by the intrinsic variation of the target (Appendix B). For a detailed discussion of this effect, see Popescu et al. (2016).

For each class and filter combination, we computed the average color, dispersion, and covariance. This allowed us to classify objects based on their distance to all the class centers, normalized by the typical spread of the class (Pajuelo 2017). This learning sample is of course limited in number, and all classes are not evenly represented. It nevertheless allowed us to estimate the Euclid capabilities by applying the classification scheme to the same sample. This is presented in Fig. 8. The leverage provided by the long wavelength coverage allowed us to clearly identify several classes: A, B, D, V, Q, and T (DeMeo et al. 2009). The main classes in the asteroid belt, the $C, S$, and $X$ (DeMeo \& Carry 2014), are more clumped, and our capabilities to classify them will depend on the exact throughput of the optical path of Euclid.

For KBOs, their spectral behavior from the blue-ish BB to the extremely red RR will place them in these graphs along a line that extends from the $\mathrm{C}, \mathrm{T}$, and $\mathrm{D}$ types (whose colors are close to those of the BB, BR, and IR classes). The RR types will be even farther from the central clump than the $D$ types. Identifying the different KBO spectral classes should therefore be straightforward with the filter set of Euclid.

In all cases, a spectral characterization using Euclid colors will benefit from the colors and spectra in the visible observed by Gaia and the LSST (Delbo et al. 2012; LSST Science Collaboration et al. 2009), the visible albedo (from IRAS, AKARI, WISE, and Herschel observations; e.g., Tedesco et al. 2002; Müller et al. 2009; Masiero et al. 2011; Usui et al. 2011), and solar phase function parameters (see 


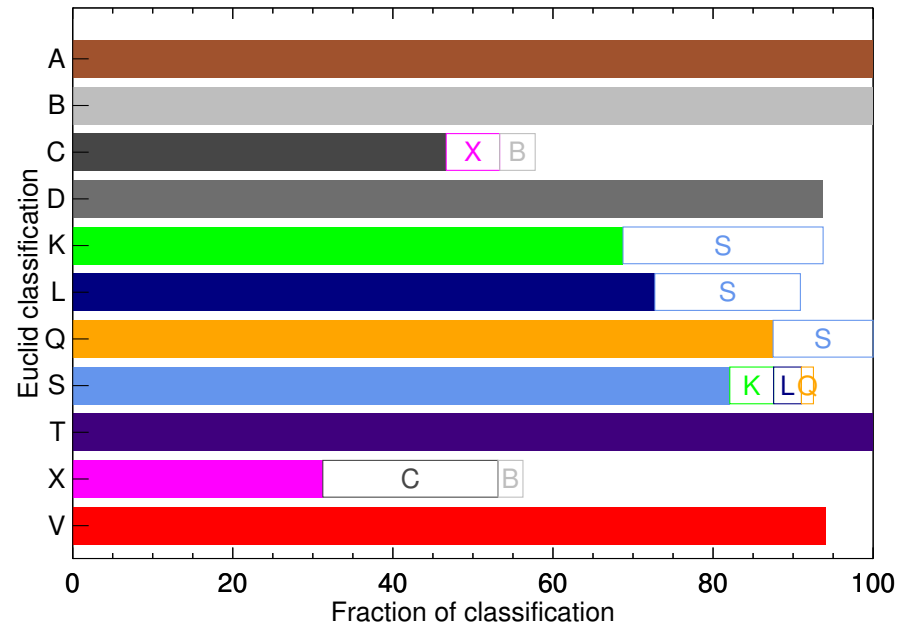

Fig. 9. Percentage of correct (solid bar) and compatible (open bar) classification for each Bus-DeMeo taxonomic classes. The Euclid photometry alone allows to classify asteroids into 11 classes.

Oszkiewicz et al. 2012, for an example of using the phase function for taxonomy). The success rate of a classification based on Euclid photometry therefore only represents a lower estimate.

We present in Fig. 9 the success rate of a classification of the 371 asteroids from the Bus-DeMeo taxonomy. The classes are generally recovered with a success rate above $60 \%$, and when misclassified, asteroids are sorted into spectrally similar (compatible) classes with a success rate closer to $90 \%$, except for the $\mathrm{C}$ and $\mathrm{X}$ classes. We did not repeat the exercise for KBOs because the available sample is limited. Because their spectral classes are very similar to those of the $\mathrm{C}, \mathrm{T}$, and D-type asteroids, and because they are even redder, we expect that it will be straightforward to identify them with the filter set of Euclid.

In summary, the VIS and NISP photometry that will be measured by Euclid seems very promising to classify SSOs into their historical spectral classes.

\section{Near-infrared spectroscopy with NISP}

Euclid will also acquire near-infrared low-resolution (resolving power of 380) spectra for many SSOs, down to $m_{\mathrm{AB}} \approx 21$, which is similar to the limiting magnitude of Gaia. Simultaneously to the four VIS exposures, NISP will acquire four slitless spectra of the same FOV. In the Wide Survey, only the red grism (1.25 to $1.85 \mu \mathrm{m})$ will be used, the usage of the blue grism $(0.92$ to $1.25 \mu \mathrm{m})$ being limited to the Deep Survey. The red grism will cover typical absorption bands of volatile compounds (e.g., water or methane ices) such as are found on distant KBOs. The main diagnostic features of asteroids (NEAs, MBAs) are located within the blue arm at $1 \mu \mathrm{m}$ and at $2 \mu \mathrm{m}$, however, which is outside the spectral range of the red grism.

Because there is no slit, many sources will be blended. To decontaminate each slitless spectrum from surrounding sources, the exposures will be taken with three different grism orientations, $90^{\circ}$ apart. For exposures whose spectral dispersion is aligned with the ecliptic, that is, which are parallel to the typical SSO motion, each SSO will blend with itself. For the remaining orientations, SSOs will often blend with background sources, which degrades both spectra. This may be a problem for the wide survey in its lowermost ecliptic latitude range, where many sources will be blended with G2V spectra from SSOs.
The apparent motion of outer SSOs being limited (Table 2), their spectra may be extracted by the Euclid consortium tools, which are designed to work on elongated sources (typically $1^{\prime \prime}$ ). Near-infrared spectra for thousands of Centaurs and KBOs could thus be produced by Euclid. It may be challenging to extract the spectra for objects in the inner solar system, and an in-depth assessment of the feasibility of such measurements is beyond the scope of this paper. In both cases, these spectra will be very similar to the low-resolution spectra that were used to define the current asteroid taxonomy (DeMeo et al. 2009) and diagnostic of the KBO class as defined by (Fulchignoni et al. 2008).

\section{Multiplicity and activity of SSOs}

With a very stable PSF and a pixel scale of $0.1^{\prime \prime}$ and $0.3^{\prime \prime}$ for VIS and NISP, which is close to the diffraction limit of Euclid, the source morphology can be studied. This is indeed one of the main goals of the cosmological survey (Laureijs et al. 2011). We first assess how Euclid might detect satellites around SSOs, and then consider their activity, that is, their dust trails.

\subsection{Direct imaging of multiple systems with Euclid}

In the two decades since the discovery of the first asteroid satellite, Dactyl around (243) Ida, by the Galileo mission (Chapman et al. 1995), direct imaging has been the main source of discovery and characterization of satellites around large SSOs in the main belt (e.g., Merline et al. 1999; Berthier et al. 2014), among Jupiter Trojans (Marchis et al. 2006, 2014), and KBOs (e.g., Brown et al. 2005, 2006, 2010; Carry et al. 2011; Fraser et al. 2017). This is particularly evident for KBOs, for which 65 of the 80 known binary systems where discovered by the Hubble Space Telescope, and the other 14 by large groundbased telescopes, often supported by adaptive optics (see, e.g., Parker et al. 2011; Johnston 2015; Margot et al. 2015). The situation is different for NEAs and small MBAs, for which most discoveries and follow-up observations were made with optical light curves and radar echoes (e.g., Pravec \& Harris 2007; Pravec et al. 2012; Fang et al. 2011; Brozović et al. 2011).

To estimate the capabilities of Euclid to angularly resolve a multiple system, we used the compilation of system parameters by Johnston (2015). We computed the magnitude difference between components $\Delta m$ from their diameter ratio, and their typical separation $\Theta$ from the ratio of the binary system semimajor axis to its heliocentric semimajor axis (Table 3).

The angular resolution of Euclid will thus allow us to detect satellites of KBOs and large MBAs, but not those around NEAs, MCs, and small MBAs. The case of KBOs is straightforward, owing to the very little smearing of their PSF from their apparent motion (Table 2). Based on the expected number of observations of KBOs (Table 1) and their binarity fraction, Euclid is expected to observe $300 \pm 200$ multiple KBO systems, which is a four-fold increase.

The case of MBAs is more complex. First, there are only 25 large MBAs with an inclination higher than $15^{\circ}$, which will make them potentially observable by Euclid. Second, the fraction and properties of multiple systems for MBAs with a diameter of between 10 and $100 \mathrm{~km}$ is terra incognita. The reason are observational biases: detection by light curves is more efficient on close-by components, and direct imaging, especially from ground-based telescopes using adaptive optics, focused on bright, hence large, primaries. If most binaries around small asteroids $(D<10 \mathrm{~km})$ are likely formed by rotational fission 
Table 3. Typical magnitude difference $(\Delta m)$ and angular separation $(\Theta)$ between components of multiple SSO systems.

\begin{tabular}{lccc}
\hline \hline Population & $\begin{array}{c}\Delta m \\
(\mathrm{mag})\end{array}$ & $\begin{array}{c}\Theta \\
\left({ }^{\prime \prime}\right)\end{array}$ & $\begin{array}{c}f \\
(\%)\end{array}$ \\
\hline NEA and MC & $1.8_{-1.8}^{+2.0}$ & $0.01_{-0.01}^{+0.01}$ & $15 \pm 5$ \\
MBA $(D<10 \mathrm{~km})$ & $2.5_{-0.9}^{+0.9}$ & $0.01_{-0.01}^{+0.01}$ & $15 \pm 5$ \\
MBA $(D>100 \mathrm{~km})$ & $5.4_{-2.7}^{+2.7}$ & $0.30_{-0.25}^{+0.25}$ & $3 \pm 2$ \\
KBO & $1.5_{-1.5}^{+2.0}$ & $0.43_{-0.43}^{+0.60}$ & $6 \pm 4$ \\
\hline
\end{tabular}

Notes. NEA and MCs share similar characteristics, and so do large MBAs and Trojans. We split MBAs into two categories according to the diameter $D$ of the main component. Estimates on the binary frequency in each populations are based on the reviews by Noll et al. (2008) and Margot et al. (2015). We only consider high-inclination KBOs here because the binary fraction in the cold belt is closer to $30 \%$ (Fraser et al. 2017).

caused by YORP spin-up (Walsh et al. 2008; Pravec et al. 2010; Walsh \& Jacobson 2015), satellites of larger bodies are the result of reaccumulation of ejecta material after impacts (Michel et al. 2001; Durda et al. 2004). Some satellites around medium-sized MBAs are therefore to be expected, but with unknown frequency. Considering a ratio of $\approx 5$ between the semimajor axis of binary system and the diameter of the main component (typical of large MBAs; see Margot et al. 2015) and the size distribution of highinclination MBAs, only a handful of potential systems would have separations that are angularly resolvable by Euclid. Finally, the apparent motion of MBAs implies highly elongated PSFs, which diminishes the fraction of detectable systems even further.

For these reasons, Euclid will contribute little if anything at all to the characterization of multiple systems among asteroids. The prospects for discovering KBO binaries are very promising, however.

\subsection{Detection of activity}

The distinction between comets and other types of small bodies in our solar system is by convention based on the detection of activity, that is, of unbound atmosphere that is also called coma. Comets cannot be distinguished based only on their orbital elements (Fig. A.1). The picture was blurred further with the discovery of comae around Centaurs and even MBAs, which are called active asteroids (see Jewitt 2009; Jewitt et al. 2015, for reviews).

The cometary-like behavior of these objects was discovered either by sudden surges in magnitude or by diffuse non-pointlike emission around them. There are currently 18 known active asteroids and 12 known active Centaurs, corresponding to $25 \mathrm{ppm}$ and $13 \%$ of their host populations, respectively. The property of the observed comae is typically 1 to 5 mag fainter than the nucleus within a $3^{\prime \prime}$ radius (although this large aperture was chosen to avoid contamination from the nucleus PSF, which extended to about $2^{\prime \prime}$ due to atmospheric seeing, Jewitt 2009).

With much higher angular resolution and its very stable PSF as required for its primary science goal (Laureijs et al. 2011), Euclid has the capability of detecting activity like this. Based on the expected number of observations (Table 1) and on the aforementioned fraction of observed activity, Euclid may observe several active asteroids and about $300_{-200}^{+300}$ active Centaurs. As in the case of multiple systems, however, the detection capability

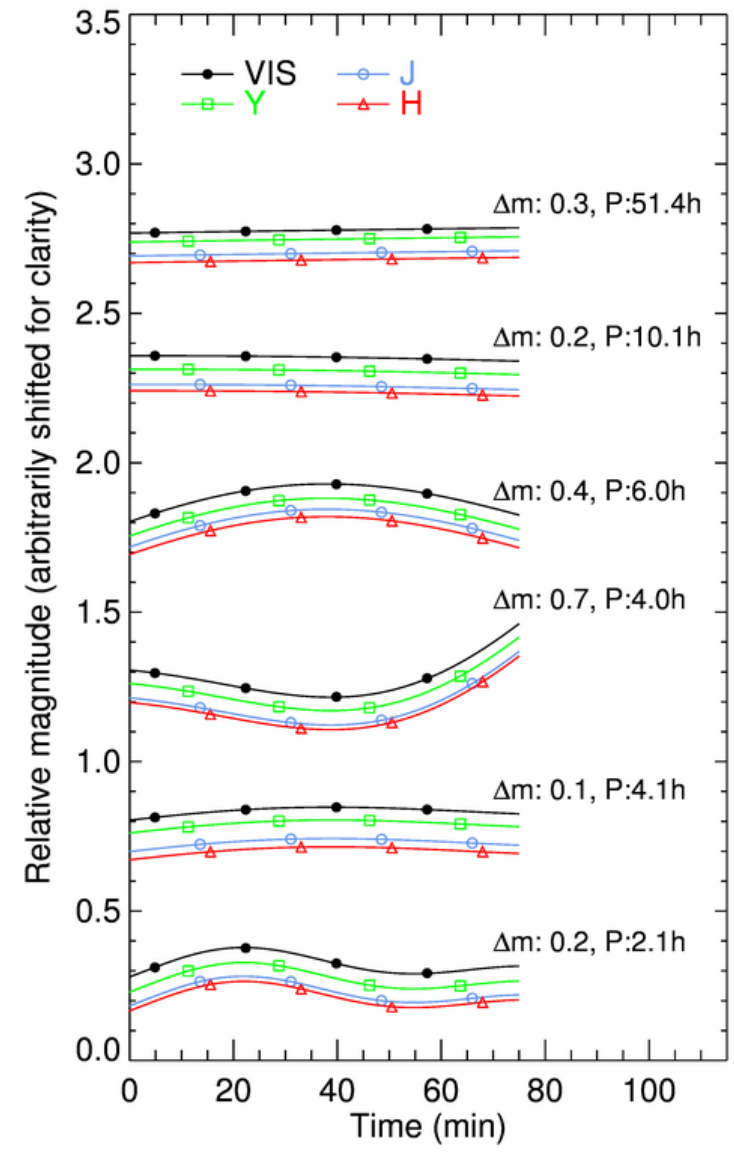

Fig. 10. Examples of simulated SSO multi-filter light curves as observed by Euclid VIS and NISP. For each light curve, the amplitude $(\Delta m)$ and rotation period $(P)$ is reported. For each, the four light curves corresponding to the different filters are printed (with a magnitude difference reduced by a factor 10 for clarity), together with the photometry at the cadence of Euclid.

will be diminished by the trailed appearance of SSOs. This will be dramatic for MBAs, but limited for Centaurs (Table 2): the typical motion will be of six pixels, that is, $0.6^{\prime \prime}$, while typical comae extend over several arcseconds.

\section{Time-resolved photometry}

The observations of each field in four repeated sequences of VIS and NISP photometry will provide hour-long light curves sampled by $4 \times 4$ measurements, or a single light curve made of 16 measurements by converting all magnitudes based on the knowledge of the SED (Fig. 10, Appendix B).

For decades, optical light curves have been the prime data set for 3D shape modeling and the study of SSO multiplicity from mutual eclipses (see the reviews by Margot et al. 2015; Durech et al. 2015). Taken alone, a single light curve, such as those that Euclid will provide, does not provide many constraints. Shape and dynamical modeling both require multiple Sun-target-observer geometries, which can only be achieved by accumulating data over many years and oppositions.

\subsection{Period, spin, and $3 D$ shape modeling}

Traditionally, the period, spin orientation, and 3D shape of asteroids were determined using many light curves that were 


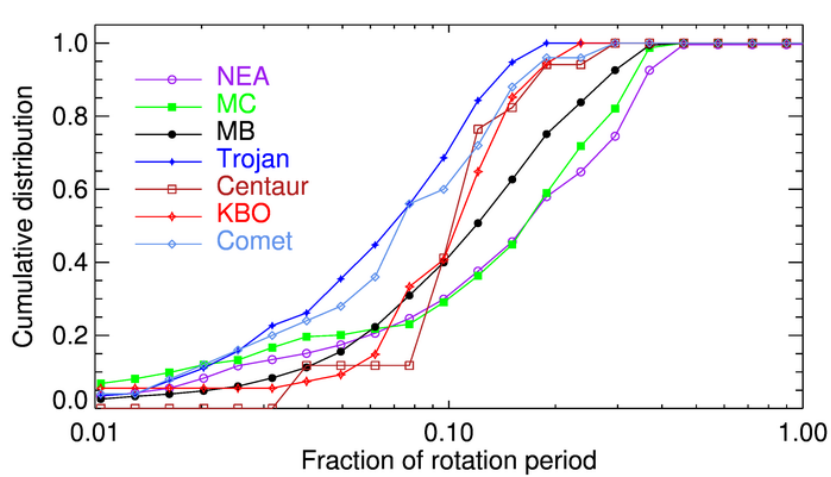

Fig. 11. Cumulative distribution of the rotation fraction covered by one hour of observations, computed on the 5759 entries with a quality code 2 or 3 from the Planetary Data System archive (Harris et al. 2017), and the 25 comets from Samarasinha et al. (2004) and Lowry et al. (2012).

taken over several apparitions (e.g., Kaasalainen \& Torppa 2001; Kaasalainen et al. 2001). It has been show later on that photometry measurements, sparse in time ${ }^{3}$, convey the same information and can be use alone or in combination with dense light curves (Kaasalainen 2004). Large surveys such as Gaia and the LSST will deliver sparse photometry for several $10^{5-6}$ SSOs (Mignard et al. 2007; LSST Science Collaboration et al. 2009).

In assessing the effect of PanSTARRS and Gaia data on shape modeling, Ďurech et al. (2005) and Hanuš \& Durech (2012) showed, however, that searching for the rotation period with sparse photometry alone may result in many ambiguous solutions. The addition of a single dense light curve often removes many aliases and harmonics in a periodogram and removes the ambiguous solutions; the effect of the single light curve depends on the fraction of the period it covers (J. Durech, pers. comm.).

The rotation periods of SSOs range from a few minutes to several hundred hours. The bulk of the distribution, however, is confined to between $2.5 \mathrm{~h}$ (which is called the spin barrier, see e.g., Scheeres et al. 2015) and 10-15 h. This implies that Euclid light curves will typically cover between 5-10 and $40 \%$ of the SSO rotation periods (Fig. 11). Euclid light curves will cover more than a quarter of the rotation (the maximum change in geometry over a rotation, used here as a baseline) for $35 \%$ of NEAs, $28 \%$ of MCs, and $16 \%$ of MBAs, and only a handful of outer SSOs. The hour-long light curves provided by Euclid will thus be valuable for 3D shape modeling of thousands of asteroids $\left(5.25_{-2.10}^{+3.50} \times 10^{3} \mathrm{NEAs}, 3.36_{-2.24}^{+4.76} \times 10^{3} \mathrm{MCS}\right.$, and $\left.1.55_{-0.35}^{+0.40} \times 10^{4} \mathrm{MBAs}\right)$.

\subsection{Mutual events and multiplicity}

Binary asteroids represent about $15 \pm 5 \%$ of the population of NEAs that are larger than $300 \mathrm{~m}$ (Sect. 7; Pravec et al. 2006), and a similar fraction is expected among MCs and MBAs with a diameter smaller than $10 \mathrm{~km}$ (Table 3; Margot et al. 2015). Most of these multiple systems were discovered by light-curve observations that recorded mutual eclipsing and occulting events (140 of the 205 binary asteroid systems known to date, the remaining are mostly binary NEAs discovered by radar echoes; see Johnston 2015).

These systems have orbital periods of $24 \pm 10 \mathrm{~h}$ and a diameter ratio of $0.33 \pm 0.17$, which implies a magnitude drop of $0.11_{-0.08}^{+0.13}$ during mutual eclipses and occultations (computed

\footnotetext{
3 Light curves whose sampling is typically longer than the period are called sparse photometry, as opposed to dense light curves, whose period is sampled by many measurements (see, e.g., Hanuš et al. 2016).
}

from the compilation of binary system properties by Johnston 2015). The hour-long light curves provided by Euclid will thus typically cover $4_{-1}^{+3} \%$ of the orbital period. When we consider that the systems are in mutual events for about $20 \%$ of the orbital period at the high phase angle probed by Euclid (e.g., Pravec et al. 2006; Carry et al. 2015), there is a corresponding probability of $\approx(5 \pm 2) \%$ to witness mutual events. Hence, Euclid could record mutual events for $900_{-450}^{+700} \mathrm{NEAs}, \mathrm{MCs}$, and MBAs, which will help to characterize these systems in combination with other photometric data sets, such as those provided by Gaia and the LSST.

\section{Conclusion}

We have explored how the ESA mission Euclid might contribute to solar system science. The operation mode of Euclid is by chance well designed for the detection and identification of moving objects. The deep limiting magnitude $\left(V_{\mathrm{AB}} \sim 24.5\right)$ of Euclid and large survey coverage (even though low ecliptic latitudes are avoided) promise about 150000 observations of SSOs in all dynamical classes, from near-Earth asteroids to distant Kuiper-belt objects, including comets.

The spectral coverage of Euclid photometry, from the visible to the near-infrared, complements the spectroscopy and photometry obtained in the visible alone by Gaia and the LSST; this will allow a spectral classification. The hour-long sequence of observations can be used to constrain the rotation period, spin orientation, 3D shape, and multiplicity of SSOs when combined with the sparse photometry of Gaia and the LSST. The high angular resolution of Euclid is expected to allow the detection of several hundreds of satellites around KBOs and activity for the same number of Centaurs.

The exact number of observations of SSOs, the determination of the astrometric, photometric, and spectroscopic precision as a function of apparent magnitude and rate, and the details of data treatments will have to be refined when the instruments are fully characterized. The exploratory work presented here aims at motivating further studies on each aspect of SSO observations by Euclid.

In summary, against all odds, a survey explicitly avoiding the ecliptic promises great scientific prospects for solar system research, which could be delivered as Legacy Science for Euclid. A dedicated SSO processing is currently being developed within the framework of the Euclid data analysis pipeline. The main goal of the mission will benefit from this addition through the identification of blended sources (e.g., stars and galaxies) with SSOs.

Furthermore, any extension of the survey to lower latitude would dramatically increase the figures reported here: there are twice as many SSOs for every $3^{\circ}$ closer to the ecliptic. Any observation at low ecliptic latitude, such as calibration fields, during idle time of the main survey or after its completion, or dedicated to a solar system survey would provide thousands of SSOs each time, allowing us to study the already-known dark matter of our solar system: the low-albedo minor planets.

Acknowledgements. The present study made a heavy use of the Virtual Observatory tools SkyBoT ${ }^{4}$ (Berthier et al. 2006, 2016), SkyBoT 3D (Berthier et al. 2008), TOPCAT ${ }^{6}$, and STILTS $^{7}$ (Taylor 2005). Thanks to the developers for their development and reaction to my requests, in particular,

\footnotetext{
4 SkyBoT: http://vo.imcce.fr/webservices/skybot/

SkyBoT 3D: http://vo.imcce.fr/webservices/skybot3d/

6 TOPCAT: http://www.star.bris.ac.uk/ mbt/topcat/

STILTS: http://wWw. star.bris.ac.uk/ mbt/stilts/
} 
J. Berthier. The present article benefits from many discussions and comments I received, and I would like to thank L. Maquet and C. Snodgrass for our discussions regarding comet properties, the ESA Euclid group at ESAC B. Altieri, P. Gomez, H. Bouy, and R. Vavrek for our discussions on Euclid and SSO, in particular P. Gomez for sharing the Reference Survey with me. Of course, I would not have had these motivating experiences without the support of the ESAC faculty (ESAC-410/2016). Thanks to F. Merlin for creating and sharing the KBO average spectra for this study. Thanks also to R. Laureijs and T. Müller for their constructive comments on an early version of this article, and to S. Paltani and R. Pello for providing the transmission curves of the VIS and NISP filters.

\section{References}

Abazajian, K., Adelman-McCarthy, J. K., Agüeros, M. A., et al. 2003, AJ, 126, 2081

Adams, E. R., Gulbis, A. A. S., Elliot, J. L., et al. 2014, AJ, 148, 55

Bannister, M. T., Kavelaars, J. J., Petit, J.-M., et al. 2016, AJ, 152, 70

Barucci, M. A., Capria, M. T., Coradini, A., \& Fulchignoni, M. 1987, Icarus, 72, 304

Bauer, J. M., Grav, T., Blauvelt, E., et al. 2013, ApJ, 773, 22

Berthier, J., Vachier, F., Thuillot, W., et al. 2006, in Astronomical Data Analysis Software and Systems XV, eds. C. Gabriel, C. Arviset, D. Ponz, \& S. Enrique, ASP Conf. Ser., 351, 367

Berthier, J., Hestroffer, D., Carry, B., et al. 2008, LPI Contributions, 1405, 8374

Berthier, J., Vachier, F., Marchis, F., Durech, J., \& Carry, B. 2014, Icarus, 239 118

Berthier, J., Carry, B., Vachier, F., Eggl, S., \& Santerne, A. 2016, MNRAS, 458, 3394

Bowell, E., Hapke, B., Domingue, D., et al. 1989, Asteroids II, 524

Bowell, E., Muinonen, K. O., \& Wasserman, L. H. 1993, in Asteroids, Comets, Meteors 1993, LPI Contributions, 810, 44

Brown, M. E., Bouchez, A. H., Rabinowitz, D. L., et al. 2005, ApJ, 632, L45

Brown, M. E., van Dam, M. A., Bouchez, A. H., et al. 2006, ApJ, 639, 43

Brown, M. E., Ragozzine, D., Stansberry, J., \& Fraser, W. C. 2010, AJ, 139, 2700

Brozović, M., Benner, L. A. M., Taylor, P. A., et al. 2011, Icarus, 216, 241

Bus, S. J., \& Binzel, R. P. 2002a, Icarus, 158, 146

Bus, S. J., \& Binzel, R. P. 2002b, Icarus, 158, 106

Carruba, V., Domingos, R. C., Nesvorný, D., et al. 2013, MNRAS, 433, 2075

Carry, B., Hestroffer, D., DeMeo, F. E., et al. 2011, A\&A, 534, A115

Carry, B., Snodgrass, C., Lacerda, P., Hainaut, O., \& Dumas, C. 2012, A\&A, 544, A 137

Carry, B., Matter, A., Scheirich, P., et al. 2015, Icarus, 248, 516

Carry, B., Solano, E., Eggl, S., \& DeMeo, F. E. 2016, Icarus, 268, 340

Carvano, J. M., Hasselmann, H., Lazzaro, D., \& Mothé-Diniz, T. 2010, A\&A, 510, A43

Chang, C.-K., Ip, W.-H., Lin, H.-W., et al. 2014, ApJ, 788, 17

Chapman, C. R., Morrison, D., \& Zellner, B. H. 1975, Icarus, 25, 104

Chapman, C. R., Veverka, J., Thomas, P. C., et al. 1995, Nature, 374, 783

Cropper, M., Pottinger, S., Niemi, S.-M., et al. 2014, in Space Telescopes and Instrumentation 2014: Optical, Infrared, and Millimeter Wave, SPIE, 9143, $91430 \mathrm{~J}$

Delbo, M., Gayon-Markt, J., Busso, G., et al. 2012, Planet. Space Sci., 73, 86

DeMeo, F., \& Carry, B. 2013, Icarus, 226, 723

DeMeo, F. E., \& Carry, B. 2014, Nature, 505, 629

DeMeo, F. E., Binzel, R. P., Slivan, S. M., \& Bus, S. J. 2009, Icarus, 202, 160

DeMeo, F. E., Binzel, R. P., Carry, B., Polishook, D., \& Moskovitz, N. A. 2014, Icarus, 229, 392

Dohnanyi, J. S. 1969, J. Geophys. Res., 74, 2531

Durda, D. D., Bottke, W. F., Enke, B. L., et al. 2004, Icarus, 170, 243

Durech, J., Grav, T., Jedicke, R., Denneau, L., \& Kaasalainen, M. 2005, Earth Moon Planets, 97, 179

Ďurech, J., Carry, B., Delbo, M., Kaasalainen, M., \& Viikinkoski, M. 2015, Asteroid Models from Multiple Data Sources (Univ. Arizona Press), 183

Eggl, S. 2011, Celes. Mech. Dyn. Astron., 109, 211

Epchtein, N., de Batz, B., Copet, E., et al. 1994, Astrophys. Space Sci., 217, 3

Fang, J., Margot, J.-L., Brozovic, M., et al. 2011, AJ, 141, 154

Fraser, W. C., Bannister, M. T., Pike, R. E., et al. 2017, Nature Astron., 1, 0088

Fulchignoni, M., Belskaya, I., Barucci, M. A., De Sanctis, M. C., \& Doressoundiram, A. 2008, The Solar System Beyond Neptune, 181

Gaia Collaboration (Prusti, T., et al.) 2016, A\&A, 595, A1

Gladman, B., Marsden, B. G., \& Vanlaerhoven, C. 2008, Nomenclature in the Outer Solar System (Univ. Arizona Press), 43

Gladman, B. J., Davis, D. R., Neese, C., et al. 2009, Icarus, 202, 104

Gladman, B., Lawler, S. M., Petit, J.-M., et al. 2012, AJ, 144, 23

Granvik, M., Morbidelli, A., Jedicke, R., et al. 2016, Nature, 530, 303

Grav, T., Mainzer, A. K., Bauer, J., et al. 2011, AJ, 742, 40
Green, J., Schechter, P., Baltay, C., et al. 2012, Wide-Field InfraRed Survey Telescope (WFIRST) Final Report, Tech. Rep.

Hanuš, J., \& Durech, J. 2012, Planet. Space Sci., 73, 75

Hanuš, J., Durech, J., Oszkiewicz, D. A., et al. 2016, A\&A, 586, A108

Harris, A. W., \& D'Abramo, G. 2015, Icarus, 257, 302

Harris, A. W., Warner, B. D., \& Pravec, P. 2017, NASA Planetary Data System

Hewett, P. C., Warren, S. J., Leggett, S. K., \& Hodgkin, S. T. 2006, MNRAS, 367,454

Holler, B. J., Milam, S. N., Bauer, J. M., et al. 2017, ArXiv e-prints [arXiv: 1709.02763]

Ivezić, Ž., Tabachnik, S., Rafikov, R., et al. 2001, AJ, 122, 2749

Ivezić, Ž., Lupton, R. H., Jurić, M., et al. 2002, AJ, 124, 2943

Jedicke, R., \& Metcalfe, T. S. 1998, Icarus, 131, 245

Jedicke, R., Larsen, J., \& Spahr, T. 2002, Asteroids III, 71

Jewitt, D. 2003, Earth Moon Planets, 92, 465

Jewitt, D. 2009, AJ, 137, 4296

Jewitt, D. C., Trujillo, C. A., \& Luu, J. X. 2000, AJ, 120, 1140

Jewitt, D., Hsieh, H., \& Agarwal, J. 2015, The Active Asteroids (Univ. Arizona Press), 221

Johnston, W. 2015, Binary Minor Planets V8.0, NASA Planetary Data System, eAR-A-COMPIL-5-BINMP-V8.0

Kaasalainen, M. 2004, A\&A, 422, L39

Kaasalainen, M., \& Torppa, J. 2001, Icarus, 153, 24

Kaasalainen, M., Torppa, J., \& Muinonen, K. 2001, Icarus, 153, 37

Laureijs, R., Amiaux, J., Arduini, S., et al. 2011, ArXiv e-prints [arXiv: 1110.3193$]$

Lazzaro, D., Angeli, C. A., Carvano, J. M., et al. 2004, Icarus, 172, 179

Lowry, S., Duddy, S. R., Rozitis, B., et al. 2012, A\&A, 548, A12

LSST Science Collaboration, Abell, P. A., Allison, J., et al. 2009, ArXiv e-prints [arXiv:0912.0201]

Maciaszek, T., Ealet, A., Jahnke, K., et al. 2014, in Space Telescopes and Instrumentation 2014: Optical, Infrared, and Millimeter Wave, SPIE, 9143, 91430K

Mahlke, M., Bouy, H., Altieri, B., et al. 2017, A\&A, in press, DOI: $10.1051 / 0004-6361 / 201730924$

Mainzer, A., Grav, T., Masiero, J., et al. 2011, ApJ, 741, 90

Marchis, F., Hestroffer, D., Descamps, P., et al. 2006, Nature, 439, 565

Marchis, F., Durech, J., Castillo-Rogez, J., et al. 2014, ApJ, 783, L37

Margot, J.-L., Pravec, P., Taylor, P., Carry, B., \& Jacobson, S. 2015, Asteroid Systems: Binaries, Triples, and Pairs, eds. P. Michel, F. E. DeMeo, \& W. F. Bottke (Univ. Arizona Press), 355

Masiero, J. R., Mainzer, A. K., Grav, T., et al. 2011, ApJ, 741, 68

McMahon, R. G., Banerji, M., Gonzalez, E., et al. 2013, The Messenger, 154, 35

Merlin, F., Hromakina, T., Perna, D., Hong, M. J., \& Alvarez-Candal, A. 2017, A\&A, 604, A86

Merline, W. J., Close, L. M., Dumas, C., et al. 1999, Nature, 401, 565

Michel, P., Benz, W., Tanga, P., \& Richardson, D. C. 2001, Science, 294, 1696

Mignard, F., Cellino, A., Muinonen, K., et al. 2007, Earth Moon and Planets, 101,97

Muinonen, K., Belskaya, I. N., Cellino, A., et al. 2010, Icarus, 209, 542

Müller, T. G., Lellouch, E., Böhnhardt, H., et al. 2009, Earth Moon Planets, 105, 209

Nesvorný, D., Jedicke, R., Whiteley, R. J., \& Ivezić, Ž. 2005, Icarus, 173, 132

Noll, K. S., Grundy, W. M., Chiang, E. I., Margot, J.-L., \& Kern, S. D. 2008, Binaries in the Kuiper Belt, eds. M. A. Barucci, H. Boehnhardt, D. P. Cruikshank, A. Morbidelli, \& R. Dotson, 345

Oszkiewicz, D. A., Bowell, E., Wasserman, L. H., et al. 2012, Icarus, 219, 283

Pajuelo, M. 2017, Ph.D. Thesis, Observatoire de Paris

Parker, A. H., Kavelaars, J. J., Petit, J.-M., et al. 2011, ApJ, 743, 1

Petit, J.-M., Bannister, M. T., Alexandersen, M., et al. 2016, in AAS/Division for Planetary Sciences Meeting Abstracts, 48, 120.16

Petit, J.-M., Kavelaars, J. J., Gladman, B. J., et al. 2017, AJ, 153, 236

Polishook, D., Ofek, E. O., Waszczak, A., et al. 2012, MNRAS, 421, 2094

Popescu, M., Licandro, J., Morate, D., et al. 2016, A\&A, 591, A115

Pravec, P., \& Harris, A. W. 2007, Icarus, 190, 250

Pravec, P., Scheirich, P., Kušnirák, P., et al. 2006, Icarus, 181, 63

Pravec, P., Vokrouhlický, D., Polishook, D., et al. 2010, Nature, 466, 1085

Pravec, P., Scheirich, P., Vokrouhlický, D., et al. 2012, Icarus, 218, 125

Rhodes, J., Nichol, B., Aubourg, E., et al. 2017

Russell, C. T., Raymond, C. A., Coradini, A., et al. 2012, Science, 336, 684

Samarasinha, N. H., Mueller, B. E. A., Belton, M. J. S., \& Jorda, L. 2004, Rotation of cometary nuclei (Univ. Arizona Press), 281

Scheeres, D. J., Britt, D., Carry, B., \& Holsapple, K. A. 2015, Asteroid Interiors and Morphology, eds. P. Michel, F. E. DeMeo, \& W. F. Bottke (Univ. Arizona Press), 745

Shankman, C., Kavelaars, J., Gladman, B. J., et al. 2016, AJ, 151, 31

Sierks, H., Lamy, P., Barbieri, C., et al. 2011, Science, 334, 487 
Skrutskie, M. F., Cutri, R. M., Stiening, R., et al. 2006, AJ, 131, 1163

Snodgrass, C., Carry, B., Dumas, C., \& Hainaut, O. R. 2010, A\&A, 511, A72

Snodgrass, C., Fitzsimmons, A., Lowry, S. C., \& Weissman, P. 2011, MNRAS, 414, 458

Spoto, F., Del Vigna, A., Milani, A., Tomei, G., \& Tanga, P. 2017, A\&A, submitted

Sykes, M. V., Cutri, R. M., Fowler, J. W., et al. 2000, Icarus, 146, 161

Szabó, G. M., Ivezić, Ž., Jurić, M., Lupton, R., \& Kiss, L. L. 2004, MNRAS, 348,987

Taylor, M. B. 2005, in Astronomical Data Analysis Software and Systems XIV, eds. P. Shopbell, M. Britton, \& R. Ebert, ASP Conf. Ser., 347, 29

Tedesco, E. F., Noah, P. V., Noah, M. C., \& Price, S. D. 2002, AJ, 123, 1056
Thomas, C. A., Trilling, D. E., \& Rivkin, A. S. 2012, Icarus, 219, 505 Usui, F., Kuroda, D., Müller, T. G., et al. 2011, PASJ, 63, 1117

Veverka, J., Robinson, M., Thomas, P., et al. 2000, Science, 289, 2088 Virtanen, J., Poikonen, J., Säntti, T., et al. 2016, Adv. Space Res., 57, 1607

Volk, K., Murray-Clay, R., Gladman, B., et al. 2016, AJ, 152, 23 Walsh, K. J., \& Jacobson, S. A. 2015, Formation and Evolution of Binary Asteroids, eds. P. Michel, F. E. DeMeo, \& W. F. Bottke, 375

Walsh, K. J., Richardson, D. C., \& Michel, P. 2008, Nature, 454, 188

Waszczak, A., Chang, C.-K., Ofek, E. O., et al. 2015, AJ, 150, 75

Wiegert, P., Balam, D., Moss, A., et al. 2007, AJ, 133, 1609

Yoshida, F., \& Nakamura, T. 2005, AJ, 130, 2900 


\section{Appendix A: Definition of small-body populations}

We describe here the boundaries in orbital elements to define the population we used thoroughout the article. The boundaries for NEA classes are taken from Carry et al. (2016), and the boundary of the outer solar system is adopted from Gladman et al. (2008).

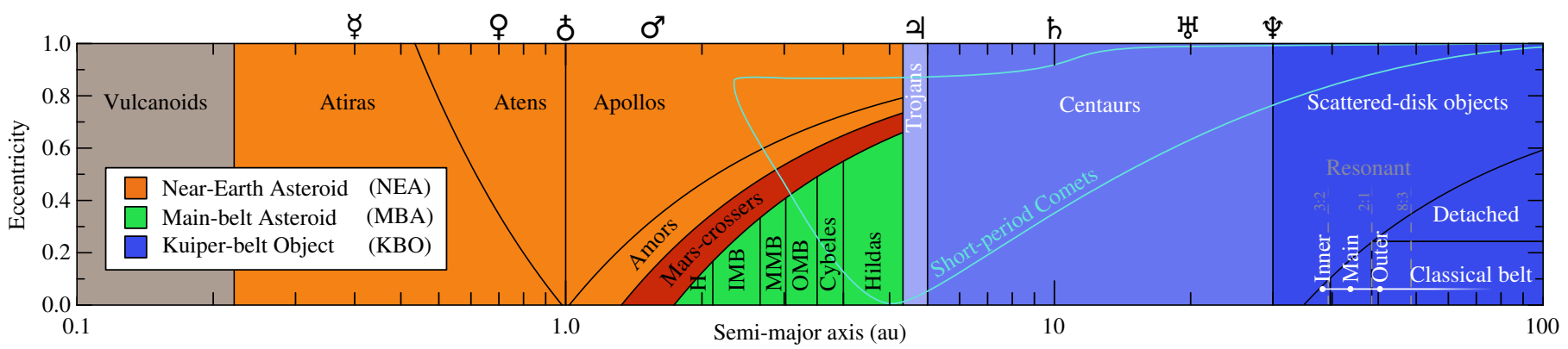

Fig. A.1. Different classes of SSOs used thoroughout the article. H stands for Hungarias, and IMB, MMB, and OMB for inner, middle, and outer belt, respectively. Comet orbital elements formally overlap with other classes because their classification is based on the presence of a coma at short heliocentric distance.

Table A.1. Definition of all the dynamical populations use here as a function of their semimajor axis, eccentricity, perihelion, and aphelion (using the definitions in Carry et al. 2016; Gladman et al. 2008).

\begin{tabular}{|c|c|c|c|c|c|c|c|c|}
\hline \multirow[t]{2}{*}{ Class } & \multicolumn{2}{|c|}{ Semimajor axis (au) } & \multicolumn{2}{|c|}{ Eccentricity } & \multicolumn{2}{|c|}{ Perihelion (au) } & \multicolumn{2}{|c|}{ Aphelion (au) } \\
\hline & min. & $\max$ & $\min$. & $\max$. & $\min$. & $\max$. & $\min$. & $\max$ \\
\hline NEA & - & - & - & - & - & 1.300 & - & - \\
\hline Atira & - & $a_{\text {f }}$ & - & - & - & - & - & $\mathrm{q}_{\dot{\delta}}$ \\
\hline Aten & - & $\mathrm{a}_{\delta}$ & - & - & - & - & $\mathrm{q}_{\dot{\delta}}$ & - \\
\hline Apollo & $a_{5}$ & 4.600 & - & - & - & $\mathrm{Q}_{\text {ठ }}$ & - & - \\
\hline Amor & $\mathrm{a}_{5}$ & 4.600 & - & - & $\mathrm{Q}_{\delta}$ & 1.300 & - & - \\
\hline $\mathrm{MC}$ & 1.300 & 4.600 & - & - & 1.300 & $\mathrm{Q}_{\sigma^{7}}$ & - & - \\
\hline MBA & $\mathrm{Q}_{\sigma^{7}}$ & 4.600 & - & - & $\mathrm{Q}_{\sigma^{\pi}}$ & - & - & - \\
\hline Hungaria & - & $\mathrm{J}_{4: 1}$ & - & - & $\mathrm{Q}_{0^{*}}$ & - & - & - \\
\hline IMB & $\mathrm{J}_{4: 1}$ & $\mathbf{J}_{3: 1}$ & - & - & $\mathrm{Q}_{0^{*}}$ & - & - & - \\
\hline MMB & $\mathbf{J}_{3: 1}$ & $\mathrm{~J}_{5: 2}$ & - & - & $\mathrm{Q}_{0^{\prime}}$ & - & - & - \\
\hline OMB & $\mathrm{J}_{5: 2}$ & $\mathbf{J}_{2: 1}$ & - & - & $\mathrm{Q}_{0^{\prime}}$ & - & - & - \\
\hline Cybele & $\mathbf{J}_{2: 1}$ & $\mathbf{J}_{5: 3}$ & - & - & $\mathrm{Q}_{0^{*}}$ & - & - & - \\
\hline Hilda & $\mathrm{J}_{5: 3}$ & 4.600 & - & - & $\mathrm{Q}_{0^{*}}$ & - & - & - \\
\hline Trojan & 4.600 & 5.500 & - & - & - & - & - & - \\
\hline Centaur & 5.500 & $\mathrm{a}_{\mathrm{O}}$ & - & - & - & - & - & - \\
\hline $\mathrm{KBO}$ & $\mathrm{aw}_{\mathrm{y}}$ & - & - & - & - & - & - & - \\
\hline SDO & $\mathrm{a}_{\mathrm{O}}$ & - & - & - & - & 37.037 & - & - \\
\hline Detached & $a_{y}$ & - & 0.24 & - & 37.037 & - & - & - \\
\hline $\mathrm{ICB}$ & $37.037^{\circ}$ & $\mathrm{N}_{2: 3}$ & - & 0.24 & 37.037 & - & - & - \\
\hline MCB & $\mathrm{N}_{2: 3}$ & $\mathrm{~N}_{1: 2}$ & - & 0.24 & 37.037 & - & - & - \\
\hline OCB & $\mathrm{N}_{1: 2}$ & - & - & 0.24 & 37.037 & - & - & - \\
\hline
\end{tabular}

Notes. See Fig. A.1 for the distribution of these populations in the semimajor axis - eccentricity orbital element space. The numerical value of the semimajor axes $a$, perihelion $q$, aphelion $Q$, and mean-motion resonances (indices ${ }_{i: j}$ ) are for the Earth $\mathrm{a}_{5}, \mathrm{q}_{5}$, and $\mathrm{Q}_{5}$ at $1.0,0.983$, and $1.017 \mathrm{AU}$; for Mars $\mathrm{Q}_{\mathrm{o}^{3}}$ at $1.666 \mathrm{AU}$; for Jupiter $\mathrm{J}_{4: 1}, \mathrm{~J}_{3: 1}, \mathrm{~J}_{5: 2}, \mathrm{~J}_{2: 1}$, and $\mathrm{J}_{5: 3}$ at 2.06, 2.5, 2.87, 3.27, $3.7 \mathrm{AU}$; and for Neptune ay , $\mathrm{N}_{2: 3}$, and $\mathrm{N}_{1: 2}$ at 30.07, 47.7, and 39.4 AU. The somewhat arbitrary limit of $37.037 \mathrm{AU}$ corresponds to the innermost perihelion that is accessible to detached KBOs (semimajor axis of $\mathrm{N}_{1: 2}$ and eccentricity of 0.24 ). 


\section{Appendix B: Euclid colors and SSO light curves}

Owing to the ever-changing Sun-SSO-observer geometry and the rotating irregular shape of SSOs, the apparent magnitude of SSOs is constantly changing. Magnitude variations in multifilter time series are thus a mixture of low-frequency geometric evolution, high-frequency shape-related variability, and intrinsic surface colors.

The slow geometric evolution can easily be taken into account (Eq. (1)), but we need to separate the intrinsic surface colors from the shape-related variability to build the SED (Sect. 5) and to obtain a dense light curve (Sect. 8). Often, only the simplistic approach of taking the pair of filters closest in time can be used to determine the color (e.g., Popescu et al. 2016), while hoping the shape-related variability will not affect the color measurements (Fig. 10, Szabó et al. 2004).

The sequence of observations by Euclid in four repeated blocks, each containing all four filters (Fig. 2), allows a more subtle approach, however. For any given color, that is, for each filter pair, each filter will be bracketed in time three times by the other filter. The reference magnitudes provided by the bracketing filter allow us to estimate the magnitude at the observing time of the other filter. For instance, to determine the (VIS-Y) index, we can use the first two measurements in VIS to estimate the VIS magnitude at the time the $Y$ filter was acquired (by simple linear interpolation for instance). This corrects, although only partially, for the shape-related variability. Hence, any colors will be evaluated six times over an hour, although not entirely independently each time.

The only notable assumption here is that the SED is constant over rotation, meaning that the surface composition and properties are homogeneous on the surface, which is a soft assumption based on the history of spacecraft rendezvous with asteroids (i.e., Eros, Gaspra, Itokawa, Mathilde, Ida, Šteins, Lutetia, and Ceres, with the only exception of the Vesta, see e.g., Veverka et al. 2000; Sierks et al. 2011; Russell et al. 2012).

We tested this approach by simulating observation sequences by Euclid. For each of the 371 asteroids of the DeMeo et al. (2009), we simulated 800 light curves made of Fourier series of the second order, with random coefficients to produce a light curve amplitude between 0 and $1.6 \mathrm{mag}$ and a random rotation period between 1 and $200 \mathrm{~h}$. These $\approx 300000$ light curves span the observed range of amplitude and period parameter space, estimated from the 5759 entries with a quality code 2 or 3 from the
Planetary Data System archive (Fig. B.1; Harris et al. 2017). We limited the simulation to second-order Fourier series as dense light curves for about a thousand asteroids from the Palomar Transient Factory showed that is was sufficient to reproduce most asteroid light curves (Polishook et al. 2012; Chang et al. 2014; Waszczak et al. 2015). For each light curve, we determined the $4 \times 4$ apparent magnitude measurements using the definition of the observing sequence of Euclid (Fig. 2) and the SSO color (from Sect. 5), and we added a random Gaussian noise of $0.02 \mathrm{mag}$.

We then analyzed these $4 \times 4$ measurements with the method described above. For each SSO and each light curve, we determined all the colors (VIS-Y, VIS-J, VIS-H, Y-J, Y-H, and J-H) and compared them with the input of the simulation, hereafter called the residuals. For each color, we also recorded the estimate dispersion.

The accuracy on each color was found to be at the level of the single measurement uncertainty (Fig. B.1). This is due to the availability of multiple estimates of each color, which improves the resulting signal-to-noise ratio. The residuals are found to be very close to zero: offsets below the millimagnitude (mmag) with a standard deviation below 0.01 , that is, smaller than individual measurement uncertainty (about a factor of five). We repeated the analysis with higher levels of Gaussian noise on individual measurements $(0.05$ and $0.10 \mathrm{mag}$, the latter corresponding to the expected precision at the limiting magnitude of Euclid), adding 600000 simulated light curves to the exercise, and found similar results: the color uncertainty remains at the level of the uncertainty on individual measurement and the residuals remain close to zero, with a dispersion following the individual measurement uncertainty reduced by a factor of about five. The colors determined with this technique are therefore precise and reliable.

The processing described here is a simple demonstration that the SED can be precisely determined from Euclid multi-filter time series. As a corollary, a single light curve of 16 measurements can be reconstructed from the $4 \times 4$ measurements. These will be the root of the spectral classification (Sect. 5) and timeresolved photometry analysis (Sect. 8). The technique will be further refined for the data processing: we considered here each color, that is, each pair of filters, independently. No attempt for a multi-pair analysis was made for this simple demonstration of the technique, while a combined analysis is expected to reduce the residuals, that is, potential biases, even more. 

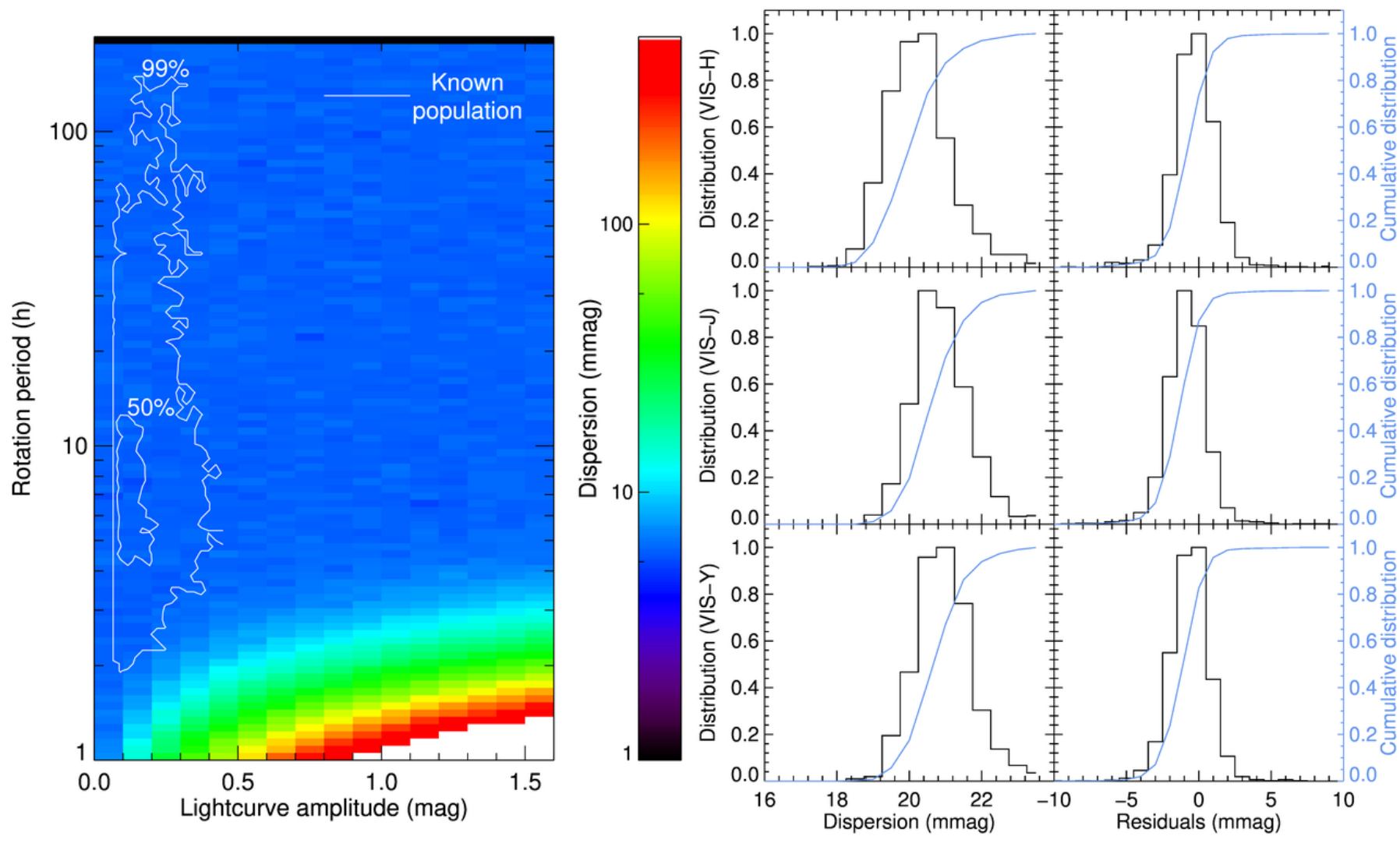

Fig. B.1. Left: distribution of the dispersion of color measurement in period-amplitude space. The white contours represent the regions encompassing 50\% and $99 \%$ of the population with known rotation period and amplitude, respectively. The largest uncertainties are found for high-amplitude short rotation-period light curves, which is outside the typical space sampled by SSOs. Right: distribution of the dispersion and residuals of color determination in VIS-Y, VIS-J, and VIS-H colors (the remaining colors are a combination of these three). The dispersion is typically at the level of the individual measurement uncertainty (here $0.020 \mathrm{mag}$ ). Residuals are much smaller, close to zero, and with a dispersion below $0.01 \mathrm{mag}$. 